\title{
Inelastic dynamic response of square membranes subjected to localised blast loading Manuscript
}

\author{
N. Mehreganian ${ }^{\mathrm{a}}$, A. S. Fallah ${ }^{\mathrm{b}}$, L.A. Louca ${ }^{\mathrm{a}}$ \\ ${ }^{a}$ Department of Civil \& Environmental Engineering, Skempton Building, South Kensington Campus, Imperial \\ College London, London SW7 2AZ, UK \\ ${ }^{b}$ Department of Mechanical and Aerospace Engineering, Brunel University London, Uxbridge UB8 3PH, UK
}

\begin{abstract}
Extensive shock and highly localised blast waves generated by detonation of near field explosives (such as Improvised Explosive Devices (IED's)) are catastrophic to structures and detrimental to humans and may result in injury or death, penetration and progressive damage, or perforation through the structure and collapse. Mitigating the effects of such waves is paramount in various aspects of design in Structural, Aeronautical, and Defense engineering, as well as being useful in Forensic Sciences. A theoretical model is presented here to predict the large inelastic deformation of ductile thin square membranes induced by a generic short duration localised pressure pulse load. The pulse loading was idealised as a multiplicative decomposition of spatial and temporal functions. The spatial part is a piecewise continuous function of axisymmetric distribution of constant pressure over a central zone of the target, then exponentially decaying beyond this zone. The temporal part may assume various shapes. Using the constitutive framework of limit analysis and incorporating the influence of finite displacements, two patterns of kinematically admissible, time dependent velocity profiles were investigated. These patterns included stationery and moving plastic hinges. The results were investigated in two cases: once with the interaction between bending moment and membrane forces retained in the analyses, and then when the response was solely governed by membrane forces.
\end{abstract}

For blast loads of high magnitude, the pressure was replaced by an impulsive velocity and the results were cast as functions of dimensionless form of initial kinetic energy. The theoretical results corroborated well with the available experimental results on high strength ARMOX steel, a class of steel impervious to the phenomenon of rate sensitivity.

Keywords: Localised blast, membrane forces, limit analysis, travelling plastic hinge, impulsive loading

\footnotetext{
* To whom correspondence should be addressed:

Email: arash.soleiman-fallah@brunel.ac.uk (Arash S. Fallah)

Tel.: +44 (0)1895266408
} 


\section{Introduction}

Mitigating the catastrophic effects of high intensity localised blasts, such as those emanating from high explosives, e.g. IED's or buried land mines, has been of prime significance in the design of protective structures over the past decades. As the blast wave momentum varies exponentially with the stand-off distance [1], near field charges give rise to localised response with much more focused energy release than those generated by distal ones, incurring large localised inelastic response and potential perforation of the structural element. As structural elements are most commonly designed as beams or plates, an assessment of the response for these elements subjected to blast waves is essential in the fields of civil, mechanical, military, and aeronautical engineering.

In fact, an extensive program of experimental and numerical studies has examined large deformation, damage evolution and failure of plates subject to uniform or localised impulsive loads. An extensive series of these studies are conducted by Nurick and co-authors [2]-[7] and by Børvik et al [8]-[11], while the main thrust of theoretical work commenced after the seminal work of Hopkinson and Prager [12]. Following their research, the dynamic plastic collapse of the rigid, perfectly plastic plates of various characteristic dimensions have been investigated [13][22]. The studies on the dynamic response of circular plates subject to rectangular pulse pressure load established that the ratio of the blast duration to the total plate response is pivotal in idealisation of the blast with zero period, i.e. uniform momentum [23], [24].

The dynamic response in terms of generalised deformations in a rigid, perfectly plastic structure is represented by evolution of plastic bending or shearing hinges. These hinges-either moving or stationery- are essentially discontinuity interfaces due to rotation (the bending hinge) or transverse shear strains (shearing hinge) leading to deformation localisations. At this weak discontinuity interfaces, the kinematic continuity of motion and the conservation of momentum must be satisfied. In thin membranes, the thickness is of a small order of magnitude compared to the characteristic in-plane lengths, the transverse shear forces may be assumed inconsequential as opposed to significant membrane forces. Thus, the deformation localisation is characterised by bending hinges only.

Langdon and Schleyer [25], [26] presented a series of numerical, analytical and experimental studies on the connection characterization and pressure pulse response of the corrugated stainless-steel blast walls. They found that the yield pressure, i.e. the pressure to cause inelastic strains at the mid-point of corrugation was reduced by increasing the flexibility of the angle connections. Nwankwo et al. [27] extended the theoretical analyses of the former authors to the CFRP (carbon fibre reinforced polymer) retrofitted blast walls using the elastic-perfectly plastic 
beam spring system with stationery bending hinges, but did ignore membrane distortions at the retrofitted section of the wall. A smaller body of literature has examined the dynamic plastic response of two-dimensional beams, shells and thin membranes [18], [28]-[30]. However, these were examined on stationery bending hinges with loading assumed to have a uniform distribution over the target; the transient phase associated with the travelling hinge has been neglected for simplicity in mathematical treatment. The purpose of this work is to examine the dynamic response of the plates with both moving and stationery bending hinges occurring due to localised blasts of various proximity of charge to the target.

\begin{tabular}{|c|c|c|c|}
\hline \multicolumn{4}{|c|}{ Notations } \\
\hline \multicolumn{4}{|c|}{ The following symbols are used in this paper: } \\
\hline \multicolumn{2}{|c|}{ Latin upper and lower case } & $r_{e}$ & Loading constant (central) zone radius $[L]$ \\
\hline$A_{i}-D_{i}$ & Integration constants; [various] & $w$ & Generalised transverse displacement; $[L]$ \\
\hline $\bar{A}$ & Elemental area; $\left[L^{2}\right]$ & $W_{\mathrm{f}}$ & Mid-point transverse displacement; $[L]$ \\
\hline$B$ & Rectangular plate side width; $[L]$ & $z$ & characteristic coordinate; [1] \\
\hline$L$ & Rectangular plate side length; $[L]$ & Greek & lower case \\
\hline$\dot{\mathcal{D}}$ & Internal energy dissipation rate; $\left[M L^{2} T^{-3}\right]$ & $\alpha$ & Impulsive velocity parameter; $\left[L^{2}\right]$ \\
\hline$\dot{E}$ & External work rate; $\left[M L^{2} T^{-3}\right]$ & $\beta$ & Static collapse pressure co-efficient; [1] \\
\hline$H$ & Plate thickness; $[L]$ & $\epsilon_{1}$ & Impulse parameter; $[L]$ \\
\hline$M$ & Plastic bending moment per unit length; $\left[M L T^{-2}\right]$ & $\varphi$ & Characteristic angle; [1] \\
\hline$M_{0}$ & Maximum plastic bending moment; $\left[M L T^{-2}\right]$ & $\eta$ & Dynamic load amplification factor; [1] \\
\hline$N$ & Plastic membrane force per unit length; $\left[M T^{-2}\right]$ & $\eta_{\text {crit }}$ & Critical dynamic load factor; [1] \\
\hline$N_{0}$ & $\begin{array}{l}\text { Maximum plastic membrane force per unit length; } \\
{\left[\mathrm{MT}^{-2}\right]}\end{array}$ & $\xi_{0}$ & Stationery plastic hinge length; [1] \\
\hline$Q$ & Transverse shear force per unit length; $\left[M T^{-2}\right]$ & $\xi(t)$ & Active travelling plastic hinge; [1] \\
\hline$T_{i}$ & Duration of the $i^{t h}$ phase; [T] & $\dot{\kappa}$ & Curvature rate; $\left[T^{-1}\right]$ \\
\hline$T_{f}$ & Final time of motion; $[T]$ & $\lambda$ & Dimensionless kinetic energy; [1] \\
\hline$V_{0}$ & Impulsive velocity of localised blast load; $\left[L T^{-1}\right]$ & $\mu$ & Areal density $(=\rho H) ;\left[M L^{-2}\right]$ \\
\hline$W_{i}$ & $\begin{array}{l}\text { Mid-point transverse displacement at the } i^{t h} \\
\text { phase; [L] }\end{array}$ & $\rho$ & Material density; $\left[M L^{-3}\right]$ \\
\hline$\dot{W}_{i}$ & $\begin{array}{l}\text { Mid-point Transverse velocity at the } i^{\text {th }} \text { phase; } \\
{\left[L T^{-1}\right]}\end{array}$ & $\dot{\theta}_{i}$ & $\begin{array}{l}\text { Rotational (angular) velocity at the outer } \\
\text { boundaries of zone i }(i=1,2) ;\left[T^{-1}\right]\end{array}$ \\
\hline$\ddot{W}_{i}$ & $\begin{array}{l}\text { Mid-point Transverse acceleration at the } i^{\text {th }} \\
\text { phase; }\left[L T^{-2}\right]\end{array}$ & $\dot{\theta_{3}}$ & $\begin{array}{l}\text { Rotational velocity across the inclined } \\
\text { plastic hinge; }\left[T^{-1}\right]\end{array}$ \\
\hline$a$ & Loading coefficient; [1] & $\sigma_{0}$ & Plastic yield/flow stress; $\left[M L^{-1} T^{-2}\right]$ \\
\hline$b$ & Loading exponent; $\left[L^{-1}\right]$ & $\sigma_{U T}$ & Static yield stress; $\left[M L^{-1} T^{-2}\right]$ \\
\hline$d_{i}$ & Ordinary Differential Equation constant; $\left[M L T^{-2}\right]$ & $\tau$ & Duration of the pulse; $[T]$ \\
\hline$p_{0}$ & Maximum overpressure; $\left[M L^{-1} T^{-2}\right]$ & $\omega_{0}$ & $r_{e} / L ;[1]$ \\
\hline$p_{c}$ & Static collapse pressure; $\left[M L^{-1} T^{-2}\right]$ & $\omega_{1}$ & Pulse factor of pattern (A); $\left[T^{-1}\right]$ \\
\hline
\end{tabular}


$p_{1}(x, y) \quad$ Spatial part of pressure pulse load; $\left[M L^{-1} T^{-2}\right] \quad \omega \quad$ Pulse factor of pattern (B); $\left[T^{-1}\right]$

$p_{2}(t) \quad$ Temporal part of pressure pulse load; [1]

Zheng et al [31] investigated the elastic-plastic performance of stiffened square plates made of Q235 low carbon steel under confined blast. The confined blast was approximated with uniform distribution, leading to global deformation of the plate, while the deformation profile was unaffected by the stiffeners and no local buckling at the interface of stiffener and the plate was observed. In all these studies shear locking was absent due to analytical formulations employed. In numerical analyses of membranes this issue will emerge and a number of techniques may be employed to eliminate or reduce its influence (e.g. Toolabi et al presented a mixed finite element formulation to enrich the shear strain and deformations of Mindlin Reissner plate [32]).

Most of the earlier studies were limited to classical theory of plates with infinitesimal deformations. The intensive shock wave may lead to large displacements in a thin plate brought about by geometry changes in contradistinction to the small deflection theory. When the deformation of the thin plate, due to a severe blast, is of a higher order of magnitude of its thickness, the structure would undergo finite displacements (geometry changes) which give rise to evolution of membrane (catenary) forces. The membrane forces so emerged will resist out-ofplane deformation and decrease its maximum at the cost of high in-plane tensile stresses. In some cases, experimental studies have revealed that in plated structures, exhibiting large deformation, the membrane forces dominate the overall performance [33]-[35]. A smaller number of theoretical research works in the literature has also catered for this phenomenon [18], [36]-[38], however, these works have considered only uniform blast. Thus, there is a paucity of information due to rarity of systematic theoretical analysis on the permanent response of plates emanating from localised blasts.

Using this rationale, this paper derives and investigates the primary features of a theoretical solution for blast loaded thin square plates. Membrane forces are introduced as a part of the solution and emerge as deformations become finite.

This paper is, as such, an extension of previous works of literature [19], [36], [37] which dealt with applying the bound theorems of plasticity to derive explicit closed form theoretical solutions which catered for the problem of dynamic response in locally blasted square plates. Following this introduction, the general assumptions made throughout the study are presented. This is followed by a discussion of the governing equations and derivation of the static plastic collapse load in Section 3. In Section 4, a rigorous analysis is conducted on the dynamic performance of plates encompassing a wide range of loading and boundary conditions. For high magnitude 


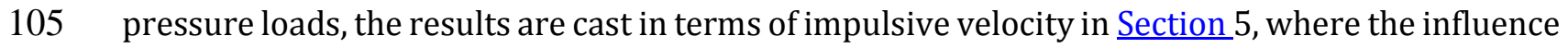

106 of boundary conditions and strain rate sensitivity is briefly studied while the theoretical results

107 are validated against available experimental and numerical results in Section 6. Finally, Section 7

108 presents and discusses the conclusions of the study.

\section{Statement of the problem}

\section{$110 \quad 2.1 \quad$ Assumptions}

111 The plates examined in this work are assumed to be 'membranes', implying that they are thin 112 enough to render the contribution of transverse shear strains and rotatory inertia negligible.

113 These effects are thus disregarded, while in-plane action plays a significant role in the overall 114 response and has been included. In fact, it has been shown that the effect of transverse shear is 115 not significant for locally blasted panels with slenderness ratio $v \geq 5$ [39]. The effect of rotatory 116 inertia is even less significant [39].

117 In order to produce reliable results for dynamic plastic response of the membranes the 118 influence of finite displacements must be retained in the study [20], [40]-[42].The overall 119 response of the structure is thus characterised by the deformed shape, rather than the base 120 configuration. This condition necessitates the membrane force $N$ to be included in the dynamic 121 energy equilibrium equations. Hence, the overall response consists of the combined effects bending and membrane phenomena.

123 The influence of visco-plasticity has been discussed in the context of the problem; however, 124 most Rolled Homogeneous Armour (RHA) graded steel types of interest in this work are 125 impervious to such phenomenological effect. Strain rate sensitivity would reduce the overall 126 deformation and increase the material strength, but in some cases reduce the rupture strain [43]. 127 A detailed theoretical procedure to determine the dynamic response of visco-plastic plates 128 subject to blasts and impacts is found in [20].

\section{2.1.1 Geometry and load characteristics}

130 An initially flat, monolithic ductile square plate with characteristic in-plane dimensions of $2 \mathrm{~L}$ 131 and thickness of $H$ is considered. The plate is secured along its periphery with simply supported 132 boundary conditions and is made of rigid-perfectly plastic material. The maximum plastic 133 moment and the maximum membrane force per unit length are denoted by $M_{0}$ and $N_{0}$, 134 respectively. It is assumed that the planar cross sections of the plate remain plane and 135 perpendicular to its neutral plane throughout deformation (Kirchhoff-Love plate's kinematic 136 assumption). 
The prescribed loading condition is of a generic blast load imparted transversely on the plated structure (Fig. 1). In most works of the literature, the loading is assumed multiplicative of spatial (load shape) and temporal (pulse shape) variations, i.e. $P(x, y, t)=p_{1}(x, y) p_{2}(t)$ (See e.g. [44][47]). The load shape, as shown by [3] maintains a uniform pressure within the central zone of radius $r_{e}$ before decaying exponentially along the radial coordinate $r$, as described in (1):

$$
\begin{gathered}
p_{1}(r)=\left\{\begin{array}{cc}
p_{0} & 0 \leq r \leq r_{e} \\
p_{0} a e^{-b r} & r_{e} \leq r \leq R
\end{array}\right. \\
p_{2}(t)= \begin{cases}1 & \text { for } 0 \leq t \leq \tau \\
0 & \text { for } t \geq \tau\end{cases}
\end{gathered}
$$

where $r=\sqrt{x^{2}+y^{2}}$ and $a=e^{b r_{e}}$ is a loading parameter. The loading radius and exponent, $r_{e}$ and $b$, respectively, may be obtained numerically through curve fitting the pressure data from commercial Finite Element software, considering the explosive type, geometry and stand off from the target. The blast load entails axisymmetric properties and thus reduces the domain of study to only one quarter of the plate.

Depending on the blast type, e.g. gas explosion or high explosive detonation, the pulse shape assumes various functions, viz. sinusoidal, triangular, exponential, linear or rectangular. While the pulse shape can have significant effect on the overall response of the dynamically loaded systems, its effect can be eliminated by utilising the Youngdahl's correlation parameters [46], [48]-[50], the efficacy of which is confirmed for monotonically decaying pulses by Ref. [42]. For impulsive blasts, the pulse shape has no intrinsic effect on the system. In this work, a rectangular pulse shape is assumed as presented in Fig. 2.

For the uniform or point loads, only the load magnitude contributes to the length of the plastic hinge lines, while in generic blasts the length depends on both the magnitude and distribution.

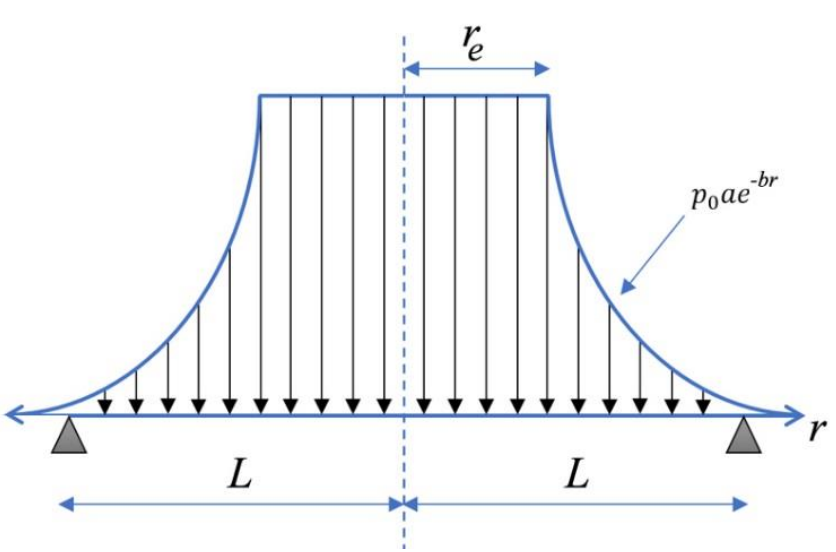

Fig. 1- Spatially constant-exponential distribution of load

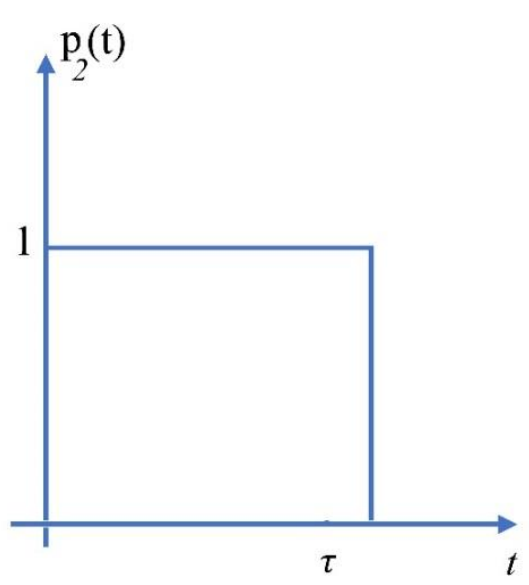

Fig. 2- Temporally rectangular pulse shape 


\subsection{Yield condition and flow rule}

158 By referring to the analysis of simply supported beams discussed in [36], and using the Tresca 159 yield condition, it may be assumed that for the simply supported plates, the plastic yield is 160 governed by the constitutive equations as follows:

$$
\begin{gathered}
\frac{N}{N_{0}}=\frac{2 w}{H},\left|M / M_{0}\right|=\left(1-\left(\frac{N}{N_{0}}\right)^{2}\right), \text { if } \frac{w}{H}<\frac{1}{2} \\
\frac{N}{N_{0}}=1, \quad \frac{M}{M_{0}}=0, \text { if } \frac{w}{H} \geq \frac{1}{2}
\end{gathered}
$$

161 For prismatic sections:

$$
N_{0}=\sigma_{0} H, \quad M_{0}=\frac{\sigma_{0} H^{2}}{4}
$$

Eq. (3) represents two parabolas plotted in Fig. 3. The state of stress and the normal to the yield surface are co-directional along the yield path of each parabola. From Eq.'s (3)-(5), it is

164 evident that $M=M_{0}$ and $N=0$ when $w=0$. Thus, the plastic flow for a perfectly rectangular 165 plate initiates at corner $\mathrm{A}$ of the yield curve, following the path $\mathrm{AB}$ of the upper right quadrant as 166 the transverse displacement increases. The exact mathematical solution is bound between the 167 two square yield surfaces (broken lines in Fig. 3) which circumscribe and inscribe the exact yield 168 condition given in ansatz Eq. (3). Therefore, the normality requirement dictates that:

$$
M=M_{0} \text { and } N=N_{0}
$$

169

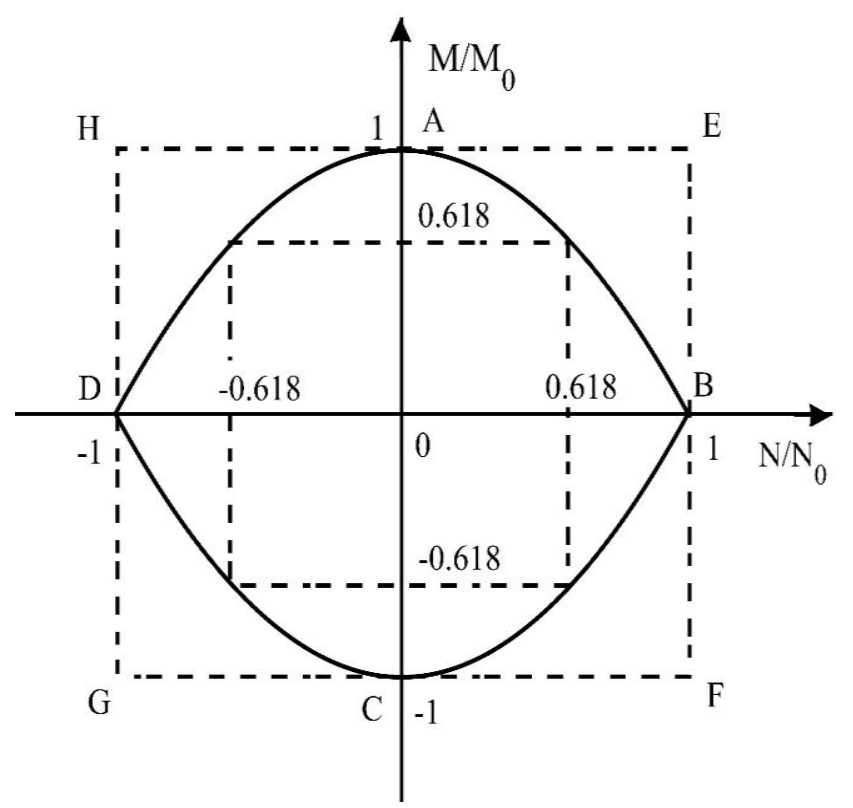


Fig. 3- Exact yield curve of the plate, vs. the circumscribing and inscribing yield curves (broken lines)

170

171

172

173

174

175

176

177

178

179

180

181

182

183

184

185

186

187

188

189

190

191

192

193

194

The assumed isotropic hardening in the constitutive formulation is accounted for by replacing the yield stress with the average of the initial yield and ultimate tensile stresses of the material. It may be possible to have a kinematic hardening formulation or a combination of isotropic and kinematic hardening to incorporate the Bauschinger effects. However, in the context of rigidperfectly plastic constitutive formulation there is no difference between the two and the simple idealisation adopted here serves the purpose.

\section{Governing Equations}

\subsection{Principle of virtual velocities}

Consider an arbitrarily shaped surface element bound by an oriented closed path in Cartesian Coordinate system. Using Green's theorem, the equation of motion in its force vector field is converted into functional of energy conservation, wherein the total internal energy rate $\dot{\mathcal{D}}$ dissipated at the continuous velocity fields, at the plastic hinges and within the plastic zones, is at equilibrium with the external work rate $\dot{E}$. For an arbitrarily shaped plate (and beams as special case) when the shear stain and rotatory inertia effects are ignored [19], [21], [36], we have:

$$
\int_{A}(p(x, y, t)-\mu \ddot{w}) \dot{w} d \bar{A}=\int_{A}(M+N w) \dot{\kappa} d \bar{A}+\sum_{m=1}^{n} \int_{A}(M+N w) \dot{\theta}_{m} d C_{m}+\sum_{u=1}^{v} Q(\dot{w})_{u} d C_{u}
$$

4 Note that the over dot notation denotes differentiation with respect to time. In Eq. (7), $\bar{A}$ is the elemental area, $\mu$ is the mass per unit surface area and $p(x, y, t)$ is the pressure field function. The expressions on the left-hand side represent the external work rate with the first term being work due to pressure field and the second term due to Lagrange-D'Alembert principle's inertia force, while the first term on the right-hand side is the strain energy dissipated in a continuous deformation field, the second term is the energy dissipated at $m$ discrete plastic hinges of length $C_{m}$, each having an angular velocity of $\dot{\theta}_{m}=\left(\partial \dot{w} / d x_{i}\right)_{m}$, where $x_{i}$ is the characteristic general coordinate in direction of the hinge line. The last term on the right-hand side is the energy dissipated in $v$ transverse shear hinges, each of length $C_{u}$ and having a velocity discontinuity of $\dot{w}_{u}$. In this work, the final term may be ignored in the analyses since the transverse shears would not intrinsically affect the response of membranes [39], [51]. 


\subsection{Static collapse pressure}

196

197

198

199

where $M_{0}$ is the maximum moment per unit length of the plate and $\beta$ is defined as:

$$
\beta=\frac{\left(b^{2} r_{e}^{2}+2 b r_{e}+2\right)}{2(L b)^{2}}-\frac{\left(b^{3} r_{e}^{3}+3 b^{2} r_{e}^{2}+6 b r_{e}+6\right)}{3 L^{3} b^{3}}+\frac{e^{-b\left(L-r_{e}\right)}(b L+2)}{(b L)^{3}}
$$

Eq. (9) may have been obtained by eliminating the inertia term in Eq. (7) and performing the integration. Fig. 4 illustrates the influence of the loading constant central zone radius on the magnitude of static collapse pressure co-efficient.

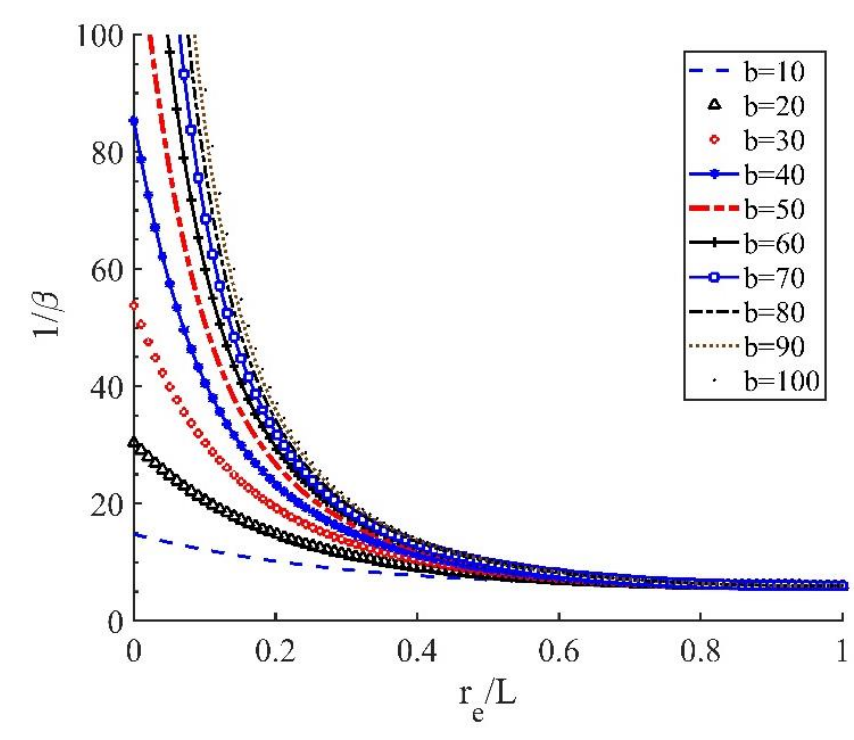

Fig. 4- Variation of the static collapse pressure co-efficient parameter with loading constant zone radius

\subsection{Deformation patterns}

The theoretical treatment presented here is in each case delineated by (i) an interactive yield surface due to combined bending and membrane action, and (ii) a yield curve where membrane 
that the hinge travels under a steady dynamic loading reflects the effect of large deflection theory [37].

Considering first a rectangular plate of length $2 L$ and width $2 B$, the current approach entails two possible velocity profiles (A) and (B) as illustrated in Fig. 5. Pattern (A) conforms to the stationery bending hinges while the length of bending hinge in pattern (B) is time dependent. It

213 has been shown, using the classical theory of circular plates [36], [46], that a critical value of the 214 load exists beyond which the static admissibility associated with stationery hinges (pattern (A))

215 is violated. For locally blasted square plate, this critical value is expressed as:

$$
1<\eta \leq\left|\frac{12 \beta}{12 \beta-1}\right|=\eta_{\text {crit }}
$$

Clearly, in the plates undergoing finite displacements, a progressively severe blast may often induce a transient phase in deformation history [18], [37]. While it may be assumed that the structures undergoing large deflections abide by the same static admissibility conditions presented above. In the limiting case of loading central zone radius $r_{e} \rightarrow L$, where the loading approaches the uniformly distributed pressure $\beta \rightarrow \frac{1}{6}$, as depicted by Eq. (10) a fact that conforms to the uniformly blast loaded square plates available in the literature [36], [37].

Thus, it may be assumed that each pattern corresponds to a special velocity profile as follows:

i. $\quad$ Profile (1): Small deflection theory, giving rise to Pattern (A), where the deformations are large enough to induce membrane forces. However, the response due to the buildup of membrane forces remains within the bounds of the critical overloading factor. Similar expression of $\eta_{\text {crit }}$ is defined by [52] for circular plates. incipient pattern (B) whose velocity profile for square plate is governed by Fig.9-Fig. 10 , which progresses into and assumes the final form of pattern (A). 


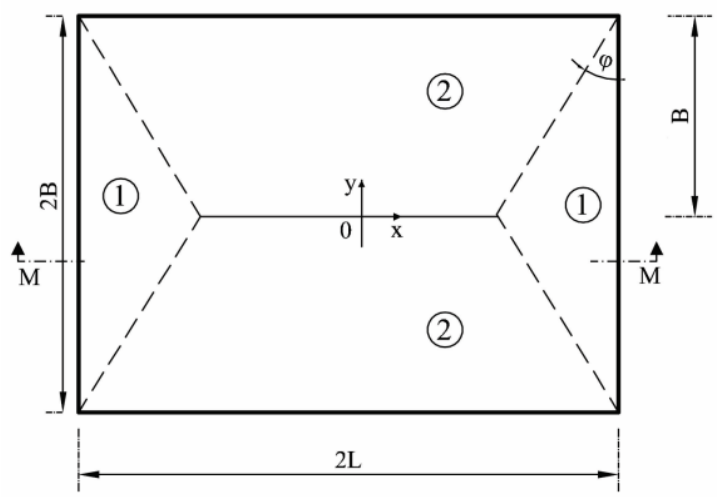

(A)

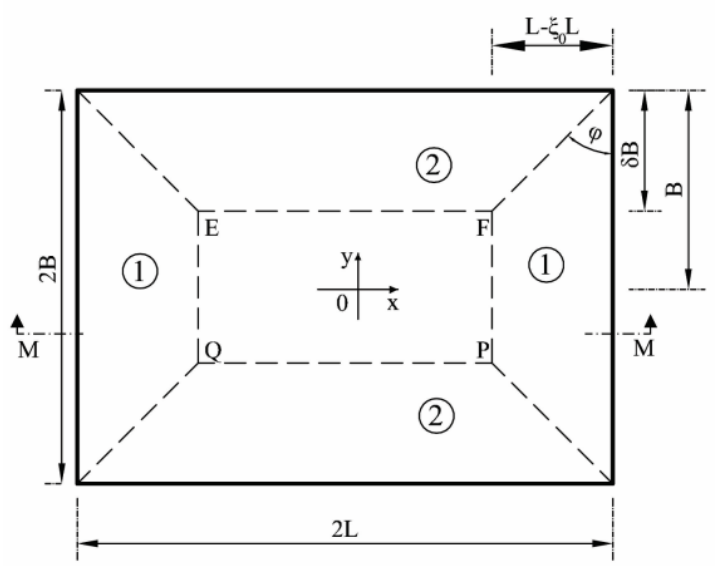

(B)

Fig. 5- Velocity profile patterns of the plate due to (A) infinitesimal transverse deflections, (B) large transverse deflections

It turns out that three distinct phases are associated with pattern (B), namely phase (i) onset of loading, phase (ii) occurrence of a transient phase with travelling plastic hinge lines which

232 move inward towards the plate centre with incipient deformation and phase (iii) the final phase 233 of deformation. The transverse velocity profile may be written in the form:

$$
\dot{w}=\frac{\dot{W}(L-x)}{L-\xi_{0} L} \quad x>\xi_{0} L
$$

In zone 1 and

$$
\dot{w}=\frac{\dot{W}(B-y)}{\delta B}
$$

In zone 2 . The velocity profiles in each zone are and kinematically admissible. The velocity profile of pattern (A) is recovered from Eq.s' (11)-(12) by choosing $\delta B=B$ and eliminating $\xi_{0}$. 237 The rotational velocities in each zone simplify to Eq.s' (13a-c), when the shearing angle at the point along the centre line of the plate is ignored, viz.:

$$
\dot{\theta}_{1}=-\frac{\partial \dot{w}}{\partial x}, \dot{\theta}_{2}=-\frac{\partial \dot{w}}{\partial y} \text { and } \dot{\theta}_{3}=\dot{\theta}_{1} \cos (\phi)+\dot{\theta}_{2} \sin (\phi)
$$

where $\dot{\theta}_{3}$ is the rotational velocity along the plastic hinge at the intersection of zone 1 and zone 2, as shown in Fig. 7. If the plate is square, $\phi=\pi / 4$ as in Fig. 5 , then both of its diagonals construct the plastic hinge lines. The dimensionless number defining the size of the rigid zone is given by $\xi_{0}$, while $\delta B=L\left(1-\xi_{0}\right)$ and $B=L$ in such plates. Thus, the velocity profile of pattern (B) would take a conical shape and recast into 


$$
\dot{w}=\frac{\dot{W}(1-z)}{\left(1-\xi_{0}\right)}
$$

which satisfies the Dirichlet boundary conditions. The auxiliary coordinate $z$ in Eq. (14) is defined for a plate of side length 2L whose diagonals lie on the Cartesian Coordinate axes as in Fig. 6. It should be noted that, while it is mathematically evident that in both cases of $b \rightarrow \infty, r_{e} \rightarrow$ 0 (i.e. the case of concentrated load) and $b \rightarrow 0, r_{e} \rightarrow L$ (i.e. the case of uniform load), the velocity profile is described by conical velocity as in Eq. (11); it is also physically reasonable to assume such a shape for the velocity profile of the rigid plate due to the localised blasts, i.e. the range of $0 \leq b<\infty$. Thus, there is no reason as to why an alternative velocity profile should exist in this range.

Given the deformation field in a rigid plated system along the equipotential surfaces has the same magnitude (for example, the path EFGH in Fig. 6 (a)), it may be assumed that the theoretical treatment with load defined by polar coordinate $(r)$ in Eq. (1) is identical to the plate in Fig. $6(\mathrm{~b})$, whose diagonals construct the plastic hinge lines. Thus, the load in the outer region is given by $p\left(z \geq r_{e} / L\right)=p_{0} a e^{-b z L}$; the range of $\mathrm{z}$ being $0 \leq z=\sqrt{x^{2}+y^{2}} \leq 1$. In fact, it is straightforward to demonstrate that the difference between energy functionals of Eq. (7) along an arbitrary polar coordinate $r$ in Fig. 6 (a) and $z$ in Fig. 6 (b) is negligible.

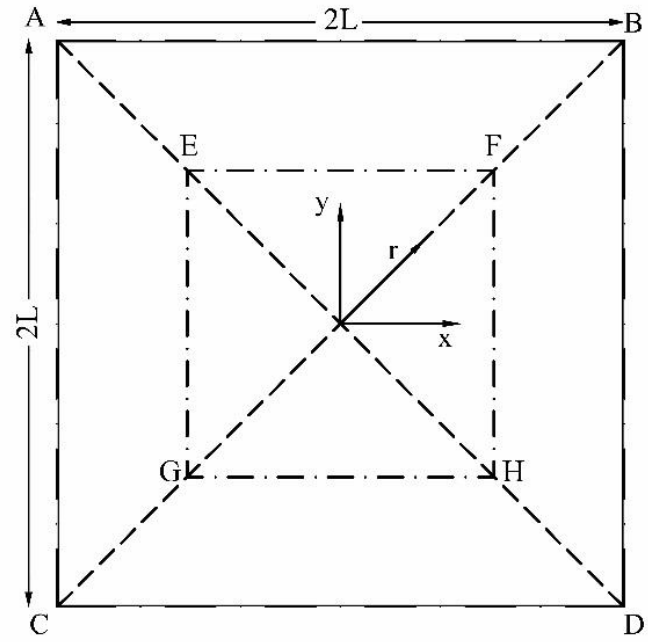

(a)

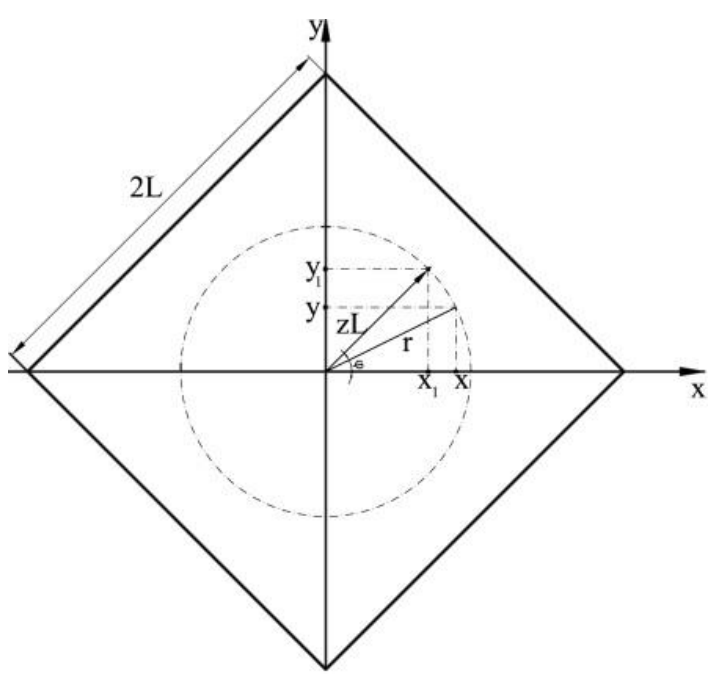

(b)

Fig. 6- (a) The top view of a simply-supported square plate (coordinates $x, y, r$ and dimensionless parameter $z$ are shown), (b) the plate with side lengths $2 \mathrm{~L}$ whose diagonals lie in the Cartesian Coordinates 


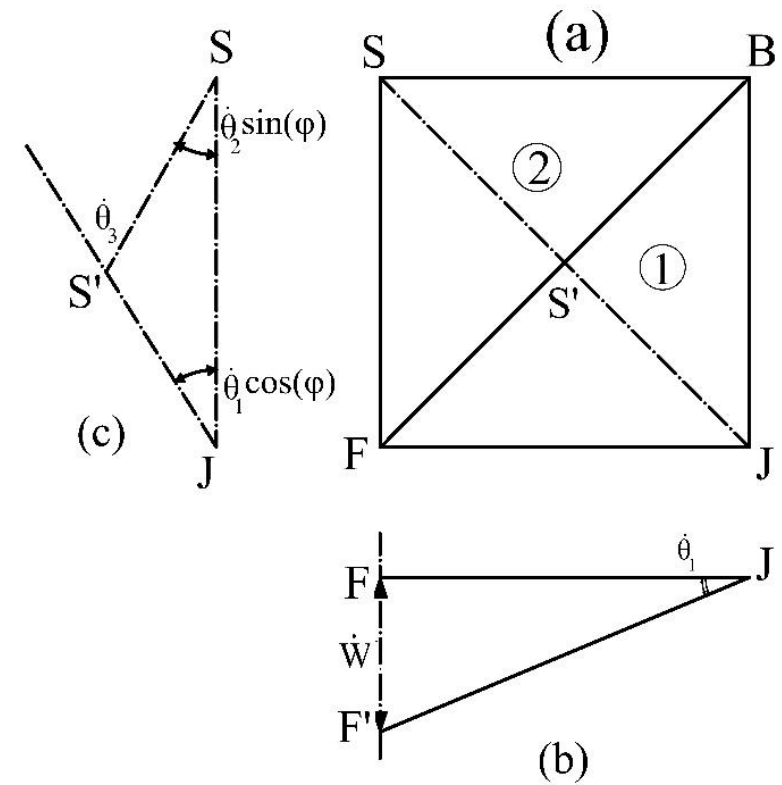

Fig. 7- Profile of the plate at intersection of zone 1 and 2. (a) top view, (b) front section (c) profile at section SJ

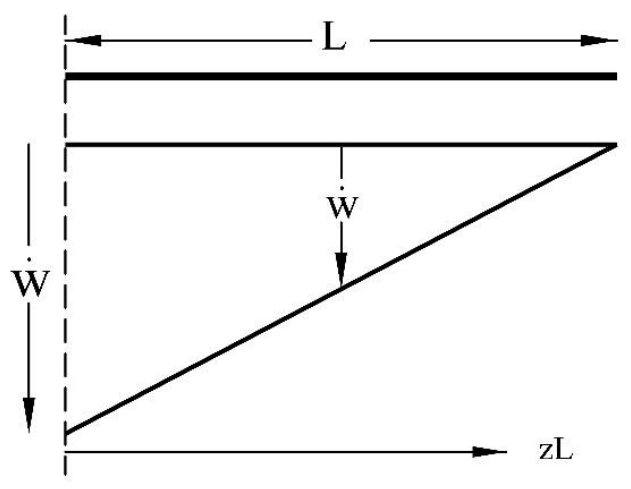

Fig. 8- Velocity profile pattern (A), (section M-M of Fig. 5)

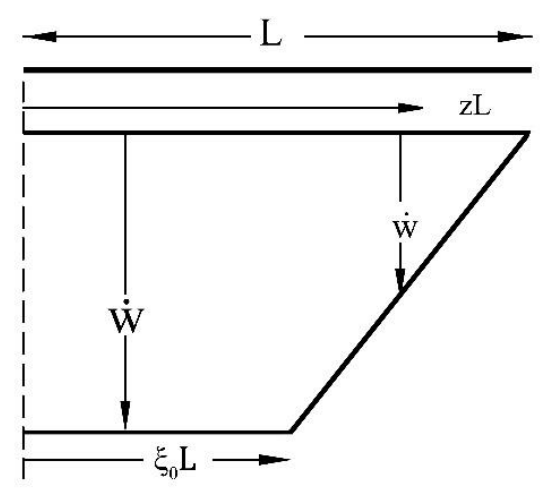

Fig.9-Velocity profile of pattern $(B)$ during the first phase of motion (section M-M of Fig. 5)

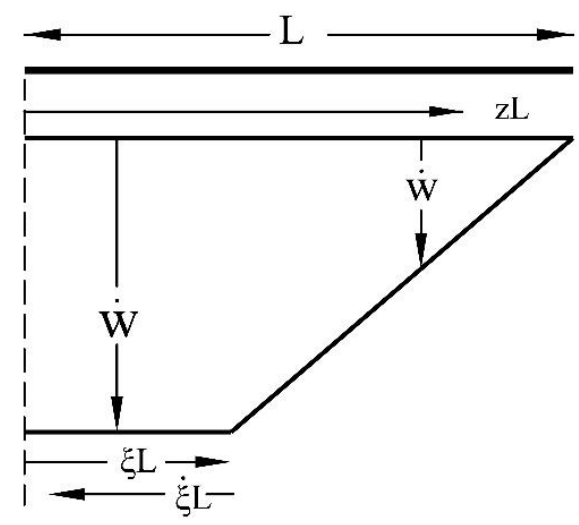

Fig. 10- Pattern (B) velocity profile during the second phase of motion 
Substituting Eq.s' (6), and (11)-(14) into Eq. (7), the internal energy rate of pattern (B) is 262 derived as:

$$
\dot{\mathcal{D}}=\frac{\left(2 H+4 W\left(1-\xi_{0}\right)\right) \dot{W} M_{0}}{H\left(1-\xi_{0}\right)}
$$

\section{Dynamic analyses}

\section{4.1 Dynamic plastic behaviour of Case 1 membranes: Pattern (A)}

265 4.1.1 Phase 1 of motion $(t \leq \tau)$

266 Regarding pattern (A), the dynamic velocity assumes a roof shape profile illustrated in Fig. 8 and identical to that due to the static loads. However, the velocity profile is now time dependent, which casts the motion into two distinct phases, i.e. phase (i) onset of loading, and phase (ii) final phase associated with the residual deformations to dissipate the reserved kinetic energy.

With reference to Fig. 3, it is assumed that the plastic flow initiates at corner $E$ of the circumscribing yield surface. The internal energy dissipation rate is distinguished from Eq. (15)

272 given $\xi_{0}=0$, while the external energy rate reads as:

$$
\dot{E}=L^{2}\left[\int_{0}^{\frac{r_{e}}{L}}\left(p_{0}-\mu \ddot{W}(1-z)\right) \dot{W}(1-z) z d z+\int_{\frac{r_{e}}{L}}^{1}\left(p_{0} a e^{-b L z}-\mu \ddot{W}\right) \dot{W}(1-z) z d z\right]
$$

By evaluating Eq. (16) and performing the integrations on the dynamic energy equilibrium equation, Eq. (3) may be recast in terms of an Ordinary Differential Equation (ODE) as:

$$
\begin{gathered}
A_{1} \ddot{W}+B_{1} W+d_{1}=0 \\
A_{1}=\frac{-1}{6} \mu L^{2} \\
B_{1}=\frac{-4 M_{0}}{H} \\
d_{1}=2 M_{0}(\eta-1)
\end{gathered}
$$

275 where $\eta=p_{0} / p_{c}$ is the load amplification factor and where the static plastic collapse is given 276 by $p_{c}=M_{0} / \beta L^{2}$. The solution of the ODE given by Eq. (17) may be written as:

$$
W_{1}(t)=\frac{1}{2} H(\eta-1)\left(1-\cos \left(\omega_{1} t\right)\right)
$$


where the subscript denotes the phase of response and $\omega_{1}=\left\{\frac{24 M_{0}}{\mu L^{2} H}\right\}^{0.5}$ hereinafter referred to 278 as the pulse factor. Clearly, $\omega_{1}^{2}=B_{1} / A_{1}$. The form of Eq. (21) may be obtained by ensuring the 279 initial boundary conditions, viz. $W_{1}(0)=0$ and $\dot{W}_{1}(0)=0$ are satisfied at the onset of loading to 280 obtain the ODE constants.

$281 \quad 4.1 .2$ Final phase of motion $(t \geq \tau)$

282 A transition from phase 1 to phase 2 occurs as the pressure load lifts off the target surface. 283 The motion is characterised by the inertia effects as $p_{0}=0$; however, the expressions (17) -(20) 284 still govern the response during the second phase of motion. Thus, by evaluating the ODE 285 parameters in Eq. (17), the transverse displacement field and its time derivatives are derived as:

$$
\begin{gathered}
\frac{\ddot{W}_{2}}{H}=\frac{\omega_{1}^{2}}{2}\left\{(\eta-1) \cos \left(\omega_{1} t\right)-\eta \cos \left(\omega_{1}(t-\tau)\right)\right\} \\
\frac{\dot{W}_{2}}{H}=\frac{\omega_{1}}{2}\left\{\sin \left(\omega_{1} t\right)(\eta-1)-\eta \sin \left(\omega_{1}(t-\tau)\right)\right\} \\
\frac{W_{2}}{H}=\frac{-1}{2}\left\{(\eta-1) \cos \left(\omega_{1} t\right)-\eta \cos \left(\omega_{1}(t-\tau)\right)+1\right\}
\end{gathered}
$$

286

287

288

289

290

291

292

The ODE solution constants are obtained through imposition of continuity conditions i.e. by imposing the same kinematic admissibility of the transverse deformation field and its time derivatives at $t=\tau$. Phase 2 terminates when the plate loses all its momentum at time $T_{2}$, which is evaluated at $\dot{W}_{2}=0$. As the analyses are conducted within the framework of rigid-plasticity rather than elasto-plasticity, no residual vibration occurs and maximum and permanent deflection fields are identical.

$$
T_{2}=\frac{1}{\omega_{1}} \arctan \left(\frac{\eta \sin \omega_{1} \tau}{1+\eta\left(\cos \left(\omega_{1} \tau\right)-1\right)}\right)
$$

Incorporating Eq. (25) in Eq. (24) yields the permanent (maximum) transverse deformation as:

$$
\frac{W_{f}}{H}=\frac{1}{2}\left(\sqrt{4\left(\eta^{2}-\eta\right) \sin ^{2} \frac{\omega_{1} \tau}{2}+1}-1\right)
$$




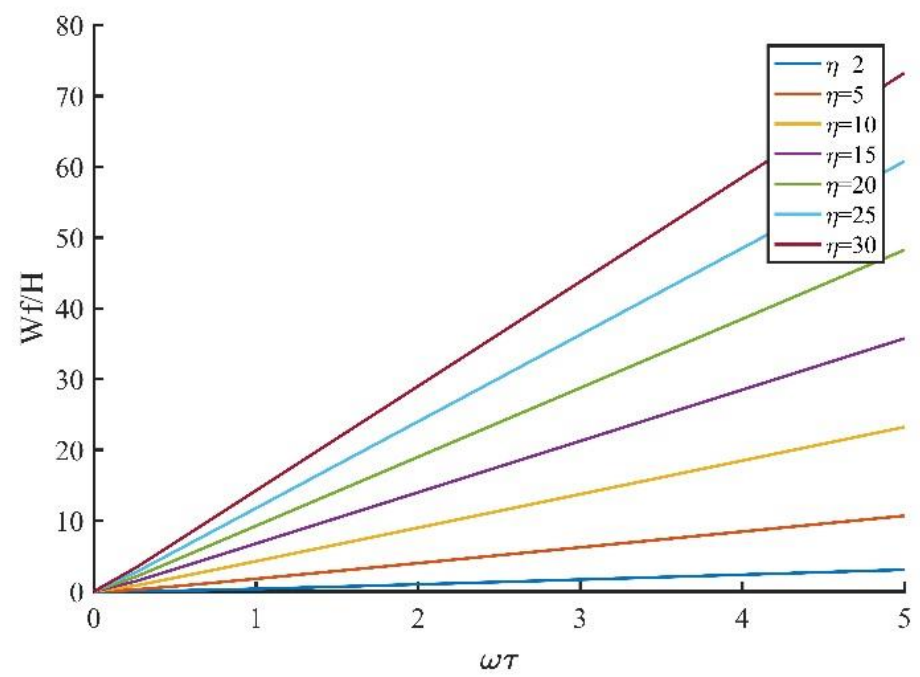

Fig. 11- Variation of the normalised permanent (maximum) deflections with $\omega_{1}$ (for interpretation of the reference to colour in the text, the reader is referred to the web version of this article)

Provided the plate (and beam as a special case) has a prismatic section, it turns out that $\omega_{1}=$ $\sqrt{\frac{6 \sigma_{0}}{\rho L^{2}}}$ depends only on the material properties $\sigma_{0}$ and $\rho$ and side length , thus its practical limits may be established. For example, given a monolithic ductile plate of $\rho=7850 \mathrm{Kg} / \mathrm{m}^{3}, \sigma_{0}$ varying between $300 \mathrm{MPa}$ for mild steel to $1210 \mathrm{MPa}$ for RHA steel, and the characteristic in-plane dimensions of prototype structures typically in the range of minimum $L=0.2 \mathrm{~m}$ to the maximum of $L=2 \mathrm{~m}$, the magnitude of $\omega_{1}$ is typically restrained as $2185 \mathrm{rad} . \mathrm{s}^{-1} \leq \omega_{1} \leq 5000 \mathrm{rad}$. $\mathrm{s}^{-1}$ for the structural elements of minimum characteristic lengths, while $\omega_{1}$ assumes the range of $218 \mathrm{rad} . \mathrm{s}^{-1} \leq \omega_{1} \leq 480 \mathrm{rad}^{-s^{-1}}$ associated with the large armour plate lengths. The pulse duration typically varies between $20 \mu$ s for localised blasts to $200 \mu$ s for distal blast loads. Hence, the range of $\omega \tau$ is restrained to values between 0.005 and 5 . The influence of this parameter against the permanent deformation is plotted in Fig. 11.

\subsection{Dynamic plastic behaviour of Case 1 membranes: Pattern (B)}

\subsubsection{First phase of motion ( $t \leq \tau)$}

In some cases, the severity of the blast load would induce the structural system to undergo a transition state in the deformation history to ensure the rate of strain energy dissipated in the plate at every instant of time equals the rate of external work done. The velocity profile in such circumstances needs to be modified, as presented in pattern (B). During the first phase; a central incipient plastic hinge of length $\left(\xi_{0}\right)$ emerges as the result of large deflections. As the blast pressure spreads throughout the structure, this plastic hinge travels toward the centre. It is further assumed that $\xi_{0}>r_{e} / L$. With reference to the velocity profile in Eq.s' (14) and (11) and Fig. 6, the total external energy rate is compiled as: 


$$
\begin{aligned}
& \dot{E}=2 L^{2}\left[\int_{0}^{\omega_{0}}\left(p_{0}-\mu \ddot{W}\right) \dot{W} z d z+\int_{\omega_{0}}^{\xi_{0}}\left(p_{0} a e^{-b L z}-\mu \ddot{W}\right) \dot{W} z d z\right. \\
& \left.+\int_{\omega_{0}}^{1}\left(p_{0} a e^{-b L z}-\frac{\mu \ddot{W}(1-z)}{1-\xi_{0}}\right) \frac{\dot{W}(1-z) z}{1-\xi_{0}} d z\right]
\end{aligned}
$$

319 where $\omega_{0}=r_{e} / L$. Evaluating the integral and performing the analysis on the energy 320 equilibrium, the expressions of the deformation fields boil down to the ODE as:

$$
A_{2} \ddot{W}+B_{2} W+d_{2}=0
$$

321 where the parameters of this expression are obtained as:

$$
\begin{gathered}
A_{2}=\frac{-1}{6} \mu L^{2}\left(3 \xi_{0}^{2}+2 \xi_{0}+1\right) \\
B_{2}=\frac{4 M_{0}\left(\xi_{0}+1\right)}{H\left(\xi_{0}-1\right)} \\
d_{2}=\frac{2 M_{0} \eta\left(\left(L b \xi_{0}+2\right) e^{-b\left(L \xi_{0}-r_{e}\right)}-(L b+2) \mathrm{e}^{-b\left(L-r_{e}\right)}+L b\left[\left(b^{2} r_{e}^{2}+2 b r_{e}+2\right)\left(\xi_{0}-1\right) / 2\right]\right)}{\beta\left(\xi_{0}-1\right) L^{3} b^{3}} \\
+\frac{2 M_{0}}{\xi_{0}-1}
\end{gathered}
$$

322 Eq. (28) is a non-homogeneous, second order ODE with constant coefficients, which has a 323 solution of the form:

$$
\begin{gathered}
W_{1}=\frac{d_{2}}{B_{2}}\{\cos (\omega t)-1\} \\
\omega=\sqrt{\frac{B_{2}}{A_{2}}}
\end{gathered}
$$

obtained through satisfying the initial conditions $W(0)=\dot{W}(0)=0$. 


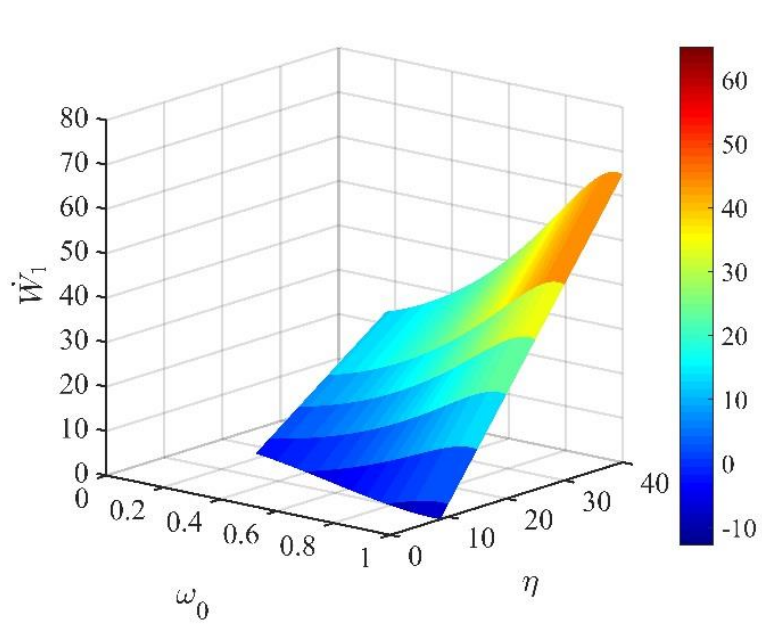

(a)

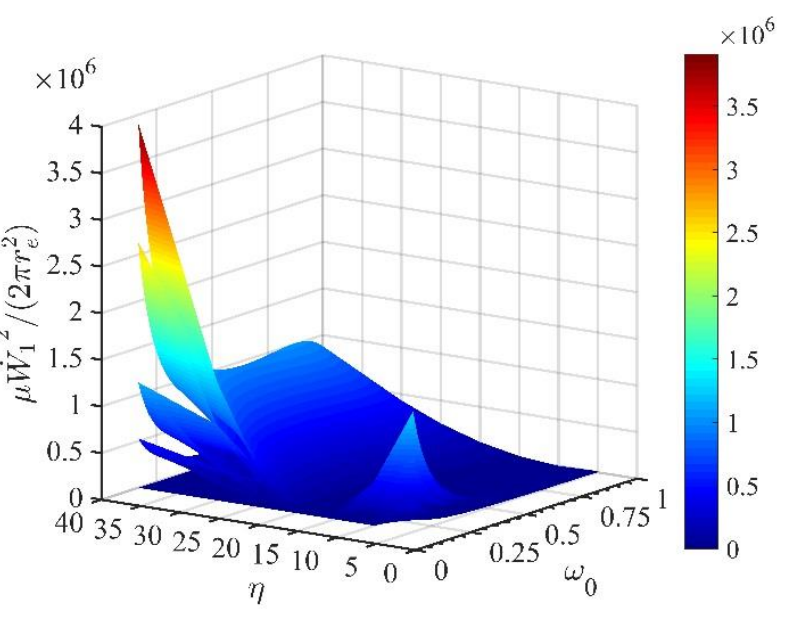

(b)

Fig. 12- The manifold of Velocity field during the first phase (a), Kinetic energy states at the end of phase 1 due to various load magnitude, where $L=150 \mathrm{~mm}, H=4 \mathrm{~mm}, \rho=7850, \sigma_{0}=1100 M P a, \xi_{0}=0.89$ (b)

The dependence of the initial velocity (and thus initial kinetic energy) on the load magnitude and radius is presented in Fig. 12. The kinetic energy is absorbed through the portion of the target that is affected by the blast load. The kinetic energy density in Fig. 12 (b) is the kinetic energy per unit area of the central blast zone. For the load radii beyond half the target length, the variation of this energy is smooth, while the blast emanating from the charge diameter to total target length of 0.25 is more plausible to cause tensile tearing, capping, and perforation through the plate. Furthermore, assuming a linear relationship between the central blast radius $r_{e}$ and the geometry of the explosive as is done by researchers [1], [53], and with a priori knowledge of the blast pressure associated with certain mass of explosive disc, the more catastrophic threat scenarios are associated with explosives of lower radii and higher charge depths than those of higher charge diameters and less depths.

Clearly, as illustrated in the Fig. 13 and Fig. 14, pulse factor is influenced by loading distribution. For proximal blasts, the pressure is concentrated at the localised region of the target centre $\left(\omega_{0} \ll 1\right)$ and decays instantly as it stretches over the target. The decay exponent of typical localised blasts varying between $50 \leq b \leq 120$ [1], [53]. The increase in the decay exponent results in reduced pulse factor but increased central deformation. In contrast, uniform pressure

341 loads assume larger values of the pulse factor as $r_{e}$ increases while $b$ decreases. Critical influence 342 of the pulse factor on the load occurs when the length of the incipient plastic hinge emerges at $3430.9^{\text {th }}$ of the characteristic plate length. Thus, it is necessary to accurately determine the position 344 of the incipient plastic hinge in the plated systems undergoing the transition state. 


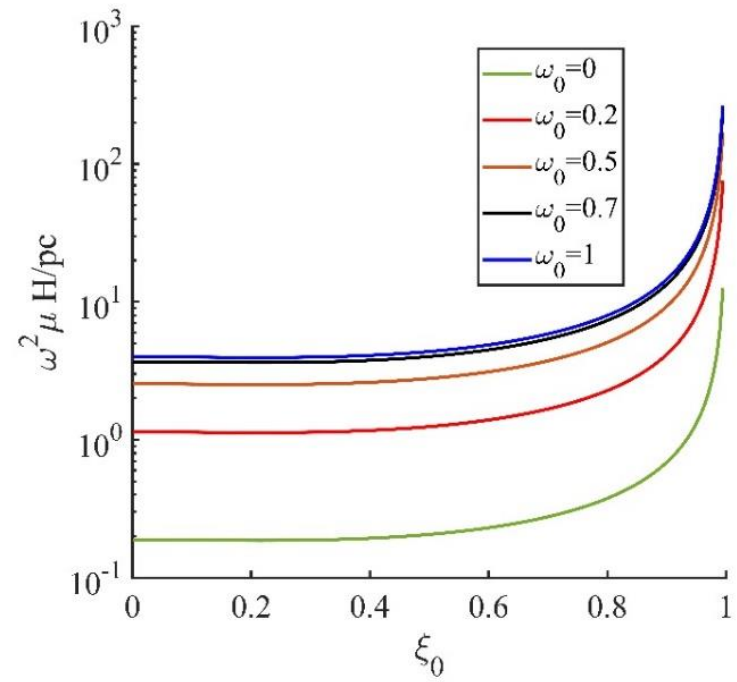

Fig. 13-Variation of the normalized pulse factor with incipient plastic hinge due to load radius

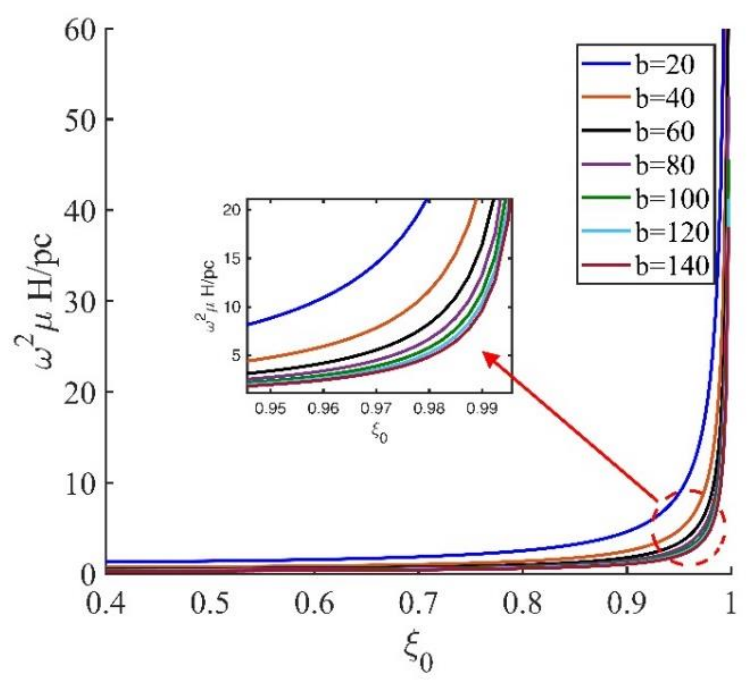

Fig. 14- - Variation of the normalized pulse factor with incipient plastic hinge due to load decay exponent

$$
f(\eta, \gamma)=\eta \gamma-12 L^{3} b^{3} \beta=0
$$

where $\eta=p_{1} / p_{c}$, and the parameter $\gamma$ is defined as:

$$
\gamma=12 a\left[b^{2} L^{2}\left(1-\xi_{0}\right) \xi_{0}+\left(1-2 \xi_{0}\right) b L-2\right] e^{-L b \xi_{0}}+12 a(L b+2) e^{-L b}-3\left(1-\xi_{0}\right)^{2}\left(\xi_{0}+1 / 3\right) L^{3} b^{3}
$$

Eq. (34) is a highly nonlinear expression and may only be solved by a numerical method. In the case of the static collapse, i.e. $\eta=1$, no real root of this equation is found, confirming that the deformation to assume is as pattern (A). This is evident as in the case of static loads pattern (A) yields a lower upper bound for static plastic collapse, thus the central plastic zone will not form in the system.

A plot of function $f$ for a range of loading parameters is presented in Fig. 15. Clearly, the length of the central zone increases with the radius of the load. In the uniform loading scenario, $\xi_{0}=1$, the incipient plastic bending hinge occurs directly at the edges. 

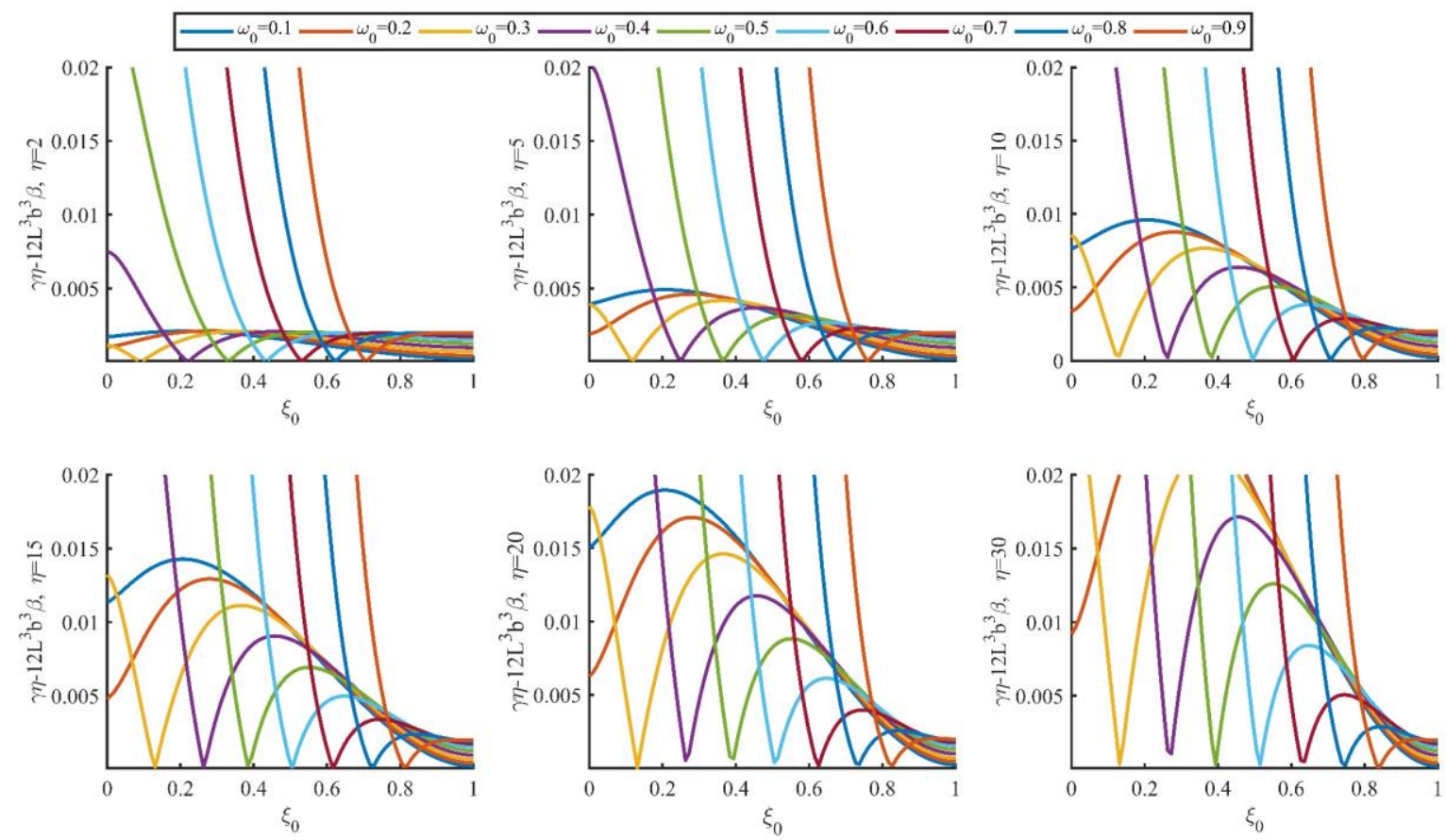

Fig. 15- Evaluating the length of central plastic bending hinge length

\subsubsection{Second phase of motion $\tau \leq t \leq T_{1}$}

363 The mathematical treatment is carried out in the same spirit to that of pattern (A). Since no

364 shearing forces at hinge lines of the central zone occurs (i.e. $Q_{i}=\frac{\partial M_{i}}{\partial i}=0$ because $M=M_{0}$, with

$365 i$ being the characteristic coordinate) and the membrane forces remain in plane parallel to the 366 initial mid-plane of the plate [37], it follows that:

$$
\mu \ddot{W}_{2}=0
$$



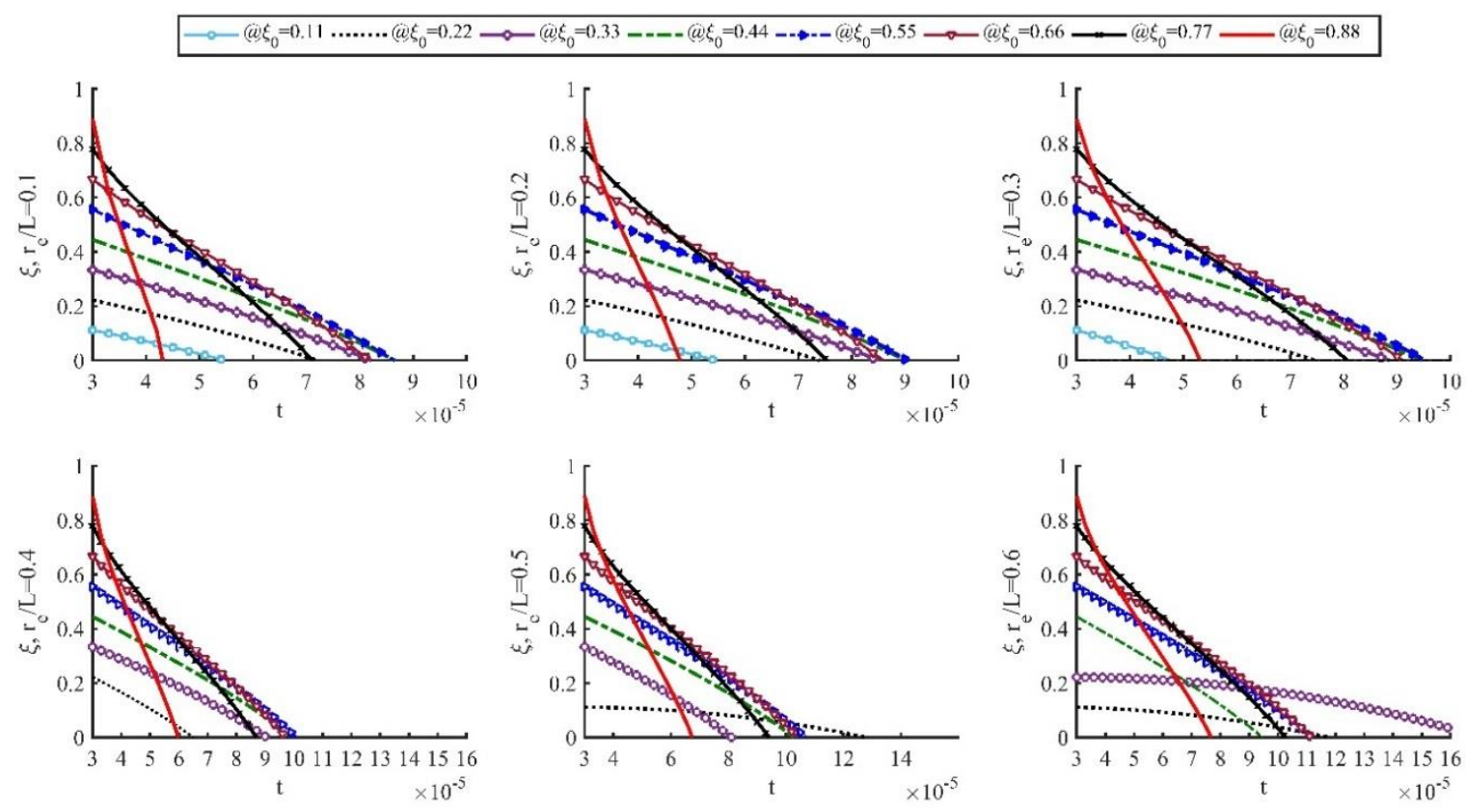

367

368

369

370

371

372

373

374

375

376

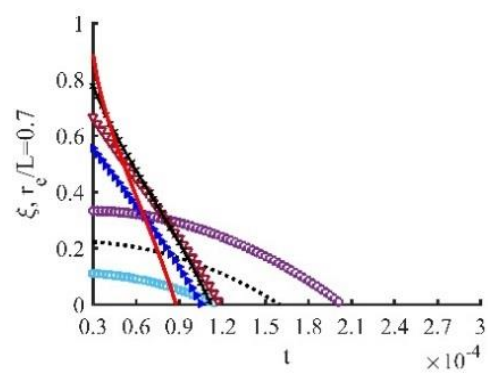
the expression
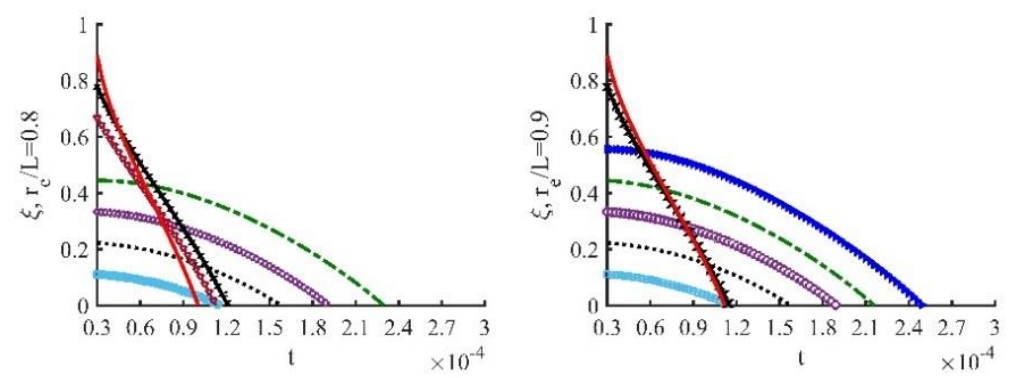

Fig.16- Determination of duration of the second phase of motion for various values of $r_{e}, b=100$ and $\eta=$ $10, \sigma_{0}=1210 \mathrm{MPa}, \mathrm{H}=4 \mathrm{~mm}$ and $L=0.2$

Thus, at the instant of sudden load removal, the potential energy in the system vanishes, but the system still maintains kinetic energy, with an associated transverse velocity field which is constant and leads the motion to continue. The incipient plastic hinge is now replaced by an active plastic hinge which is time dependent and travels inward. The occurrence of this bending hinge is attributed to the reduction in the dissipated energy. Provided $\xi \geq r_{e} / L$, the external energy rate is composed of the components of the convective derivative of transverse velocity field, given by

$$
\begin{aligned}
\dot{E}=2 L^{2}\left[\int _ { 0 } ^ { \omega _ { 0 } } \left(p_{0}\right.\right. & \left.-\mu \ddot{W}_{2}\right) \dot{W}_{2} z d z+\int_{\omega_{0}}^{\xi}\left(p_{0} a e^{-b L z}-\mu \ddot{W}_{2}\right) \dot{W}_{2} z d z \\
& \left.+\int_{\xi}^{1}\left(p_{0} a e^{-b L z}-\frac{\mu \ddot{W}_{2}(1-z)}{1-\xi}-\frac{\mu \dot{W}_{2} \dot{\xi}(1-z)}{(1-\xi)^{2}}\right) \dot{W}_{2} z\left(\frac{1-z}{1-\xi}\right) d z\right]
\end{aligned}
$$

where $\omega_{0}=r_{e} / L$ and the terms of transverse inertia may be eliminated to yield $\dot{E}=$ $-\frac{1}{2} \dot{W}_{2}^{2}\left(\xi+\frac{1}{3}\right) \dot{\xi} L^{2} \mu$. The size of the central zone decreases monotonically while moving transversely and ultimately vanishes at time $T_{1}$. A time integration of the Eq. (36) yields the 
maximum plastic transverse deformations (Eq.'s (38),(39)). The constants of integration are obtained by ensuring the kinematic conditions of displacement and velocity fields at the instant of load completion, while the constants $A_{2}, B_{2}$ and $d_{2}$ are expressed previously in Eq. (29)-(31).

$$
\begin{gathered}
\mu \dot{W}_{2}=\frac{-d_{2} \sin (\omega \tau)}{\omega A_{2}} \\
\mu W_{2}=\frac{d_{2}}{\omega^{2} A_{2}}[\omega \sin (\omega \tau)(\tau-t)+\cos (\omega \tau)-1]
\end{gathered}
$$

Eq.'s (36)- (39) should satisfy the constitutive equation of motion and the energy equilibrium outlined in Section 3.1. With reference to Eq. s' (37) and (15) and by using Eq.'s (38), and (39), Eq. (36) is recast an ODE as:

$$
\begin{aligned}
f(t, \xi, \dot{\xi})=\frac{1}{6}\left(\frac{\mu L^{2} d_{2}}{A_{2} \omega}\right)(3 \xi+1)(\xi-1) \dot{\xi}+2 M_{0} \\
-\frac{4 M_{0} d_{2}(\xi+1)(\omega \sin (\omega \tau)(t-\tau)-\cos (\omega \tau)+1)}{A_{2} \omega^{2} H}=0
\end{aligned}
$$

386
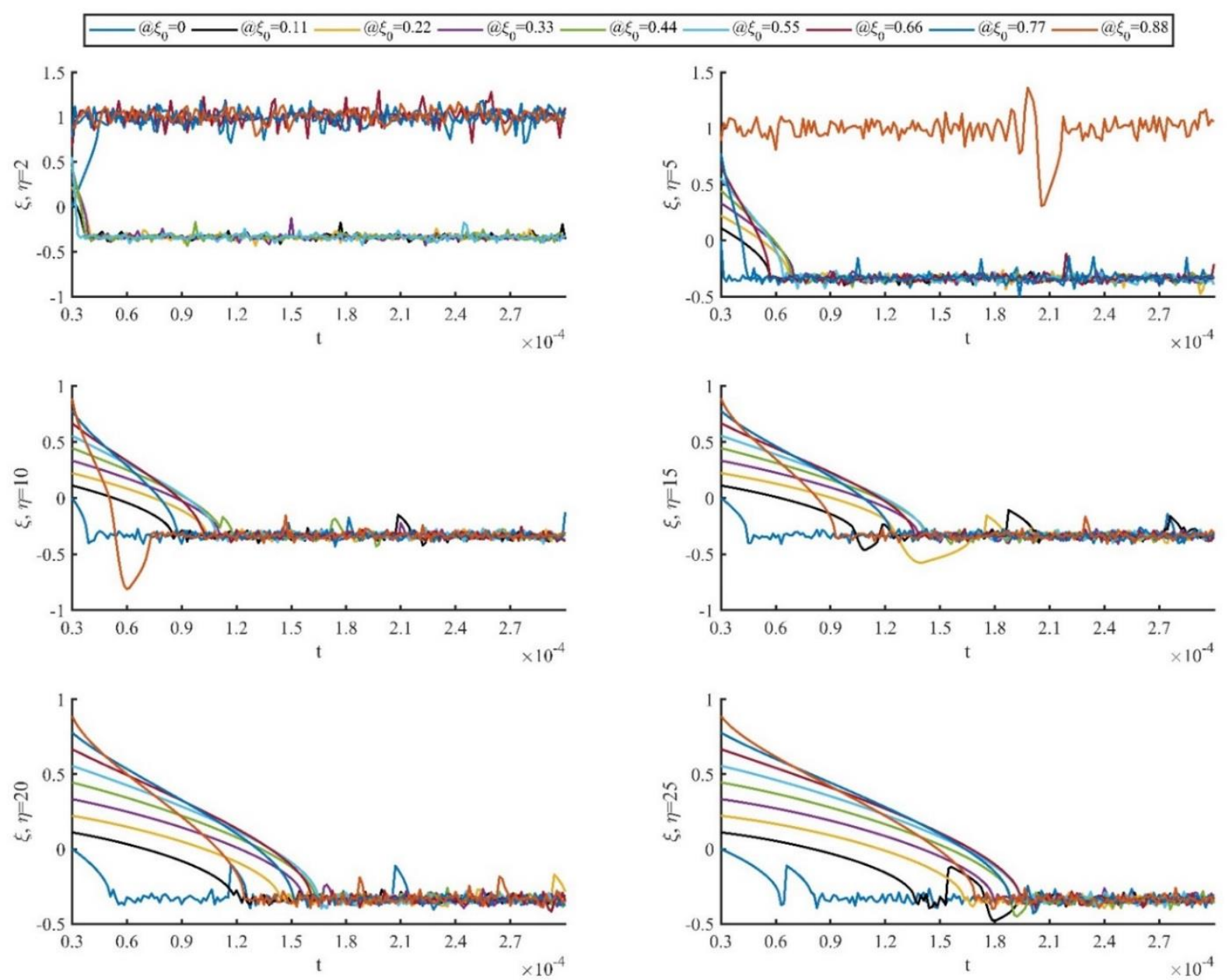

Fig. 17- Determination of duration of the second phase $T_{2}$ of motion for various values of load dynamic amplification factor, $\sigma_{0}=1210, H=4 \mathrm{~mm} b=100 \omega_{0}=0.12$ 
400

401

402

403

404

405

406

407

408

409

410

This equation is solved numerically by $5^{\text {th }}$ order Runge-Kutta method embedded in MATLAB ${ }^{\circledR}$, whereby the length of the active plastic hinge at any time is determined. The solutions to the ODE for various values of $\xi_{0}$ are plotted in Fig.16-Fig. 17. The blast duration of $\tau=30 \mu$ s for various load amplification factors is assumed. Indeed, the duration of this phase is evaluated when the ODE solution satisfies $\xi(t)=0$. In the circumstances where no real solution is attained, commonly occurring when $\omega_{0} \ll 1$ and $\xi_{0} \ll 1$, the response is governed by pattern (A) of motion.

While it may not be straightforward to investigate the static admissibility of pattern (B), it is evident that Eq.s' (32), (36)-(39) are kinematically admissible. The theoretical solution is exact when the generalised stress field is statically admissible and the associated velocity field is kinematically admissible.

\subsection{Third Phase of Motion $\mathrm{T}_{2}<\mathrm{t}<\mathrm{T}_{3}$}

Phase 2 terminates as the in-plane motion of the plastic hinge line ceases at time $T_{2}$. Now, due to the reserved kinetic energy remaining from the previous deformation, the inertia is induced which marks a transition to phase 3 , until all the residual kinetic energy is dissipated before the plate rests. Thus, the plate profile develops from pattern (B) to that of the pattern (A) with inertia term determined in Eq.(22). The expressions of the transverse velocity and the succeeding transverse deformation are recovered from successive time integrations of Eq.(22). The integration constants in these expressions are found by ensuring the kinematic continuity conditions at $t=T_{2}$. The final form of the deformation fields may be written as in Eq.'s (41) and (42).

$$
\begin{aligned}
& \frac{\dot{W}_{3}}{H}=\frac{\omega_{1}}{2}\left\{\eta\left[\sin \left(\omega_{1}\left(T_{2}-\tau\right)\right)-\sin \left(\omega_{1}(t-\tau)\right)\right]+(\eta-1)\left(\sin \left(\omega_{1} \mathrm{t}\right)-\sin \left(\omega_{1} T_{2}\right)\right)\right\} \\
& -\frac{d_{2} \sin (\omega \tau)}{A_{2} \omega \mathrm{H}} \\
& \frac{W_{3}}{H}=\frac{1}{2} \omega_{1}\left(T_{2}-t\right)\left\{(\eta-1) \sin \left(T_{2} \omega_{1}\right)-\eta \sin \left(\omega_{1}\left(T_{2}-\tau\right)\right)\right\} \\
& +\frac{\eta}{2}\left\{\cos \left(\omega_{1}(t-\tau)\right)-\cos \left(\omega_{1}\left(T_{2}-\tau\right)\right)\right\} \\
& +(\eta-1)\left[\cos \left(T_{2} \omega_{1}\right)-\cos \left(\omega_{1} t\right)\right]+\frac{d_{1} \sin (\omega \tau)}{A_{1} \omega H}\left(T_{2}-t\right) \\
& -\frac{d_{1}\left\{\omega \sin (\omega \tau)\left(T_{2}-\tau\right)+1-\cos (\omega \tau)\right\}}{A_{1} \omega^{2}}
\end{aligned}
$$

411 When $\dot{W}_{3}=0$ the plate rests. Defining $\Gamma$ in Eq. (43), an expression of the final time of the 412 deformation is delineated as per Eq. (44). 


$$
\begin{gathered}
\Gamma=\frac{1}{2} H \omega_{1} \sin \left(T_{2} \omega_{1}\right)(\eta-1)-\frac{1}{2} H \eta \omega_{1} \sin \left(\omega_{1}\left(T_{2}-\tau\right)\right)+\frac{d_{2} \sin (\omega \tau)}{A_{2} \omega} \\
T_{F}=-\frac{i}{\omega} \ln \left(\frac{2 i \Gamma e^{\frac{1}{2} i \omega \tau} \mp e^{\frac{1}{2} i \omega \tau} \sqrt{\left(-H^{2} \omega^{2} \eta(\eta-1)\left(e^{2 i \omega \tau}+1\right)+\left(\left(2 \eta^{2}-2 \eta+1\right) H^{2} \omega^{2}-\Gamma^{2}\right) e^{i \omega \tau}\right)}}{\omega H\left((\eta-1) e^{i \omega \tau}-\eta\right)}\right)
\end{gathered}
$$

413 Under the impulsive loading conditions, the expression of the full form permanent 414 deformation in Eq. (42) may be further reduced by assuming the deformation time of phase 1 and 4152 are infinitesimal such that $\sin \omega \tau \cong \omega \tau, 1-\cos \omega \tau \cong \omega^{2} \tau^{2} / 2, \sin \omega T_{2} \cong \omega T_{2}$ and $1-\cos \omega T_{2} \cong$ $416 \omega^{2} T_{2}^{2} / 2$. These assumptions are pertinent to the most cases of localised blasts generated by high 417 explosives, where the loading duration is infinitesimal ( $\tau \leq 50 \mu s)$ compared to the natural period 418 of the structure, due to the short stand-off, the reflected pressure induced by the wave front is 419 dissipated in a shorter timeframe than in distal blasts. The Full form of the Eq. (42) may be used 420 for higher accuracy or the longer loading duration is required, in which case the response of the 421 plate may be idealised as quasi-static/dynamic load. In Fig. 18 the dependence of the permanent 422 deformation on the pulse factor $\omega$ is plotted.

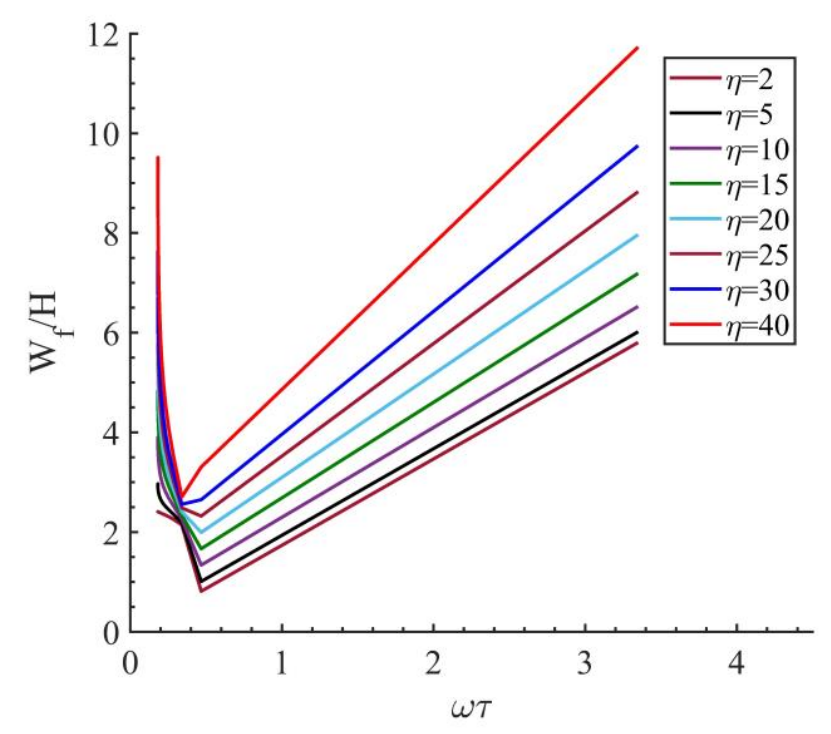

Fig. 18- $L=150 \mathrm{~mm}, H=4 \mathrm{~mm}, \rho=7850, \sigma_{0}=$ $1100 M P a$, variation of the normalised permanent deflections with $\omega$

It is appreciated that obtaining an explicit form of the permanent deformation is fraught with difficulty, due to the interdependence of the load parameters, duration of each phase and the plastic hinge lengths. While the increased number of variables makes attaining the exact theoretical solution cumbersome, particularly when the visco-plasticity and hardening effects are 
obtained by neglecting the bending moment contribution in the overall response of the plate as follows.

\subsection{Simplification into a membrane}

High intensity shock loads may give rise to large plastic deformations, to the extent that the influence of finite displacements due to such loads governs the overall response of the structure. In such cases, the contribution of the membrane forces alone associated with the finite displacements transcends those of the bending moments and transverse shear effects especially for thick plates. This leads to further simplification of the energy equilibrium expressions as the plastic energy due to bending moment may be essentially neglected; and the membrane forces solely govern the response throughout the motion. Thus, the ansatz Eq. (7) boils down to

$$
\int_{A}(p(x, y, t)-\mu \ddot{w}) \dot{w} d \bar{A}=\int_{A} N w \dot{\kappa} d \bar{A}+\sum_{m=1}^{n} \int_{A}(N w) \dot{\theta}_{m} d C_{m}
$$

the mathematical approach introduced herein is identical to that outlined in Section 4.1, while the terms of $M_{0}$ from the internal energy rate vanish. With this simplification in mind, the mathematical treatment is carried out for both patterns of the velocity profile hereunder.

\subsubsection{Pattern (A) of motion}

In the first phase of motion, Eq. (7) reduces to

$$
\ddot{W}_{1}+\omega_{1}^{2} W_{1}+d_{3} / \mathrm{A}_{1}=0
$$

where $d_{3}=2 M_{0} \eta ; A_{1}, \omega_{1}$ defined in Section 4.1.1. The ODE of Eq. (46) has a general solution as $W_{1}=C_{1} \sin \left(\omega_{1} t\right)+C_{2} \cos \left(\omega_{1} t\right)-\frac{d_{3}}{\omega_{1}^{2} A_{1}}$, the constants thereof are determined by ensuring the kinematic conditions at $t=0$, giving:

$$
\frac{W_{1}}{H}=\frac{\eta}{2}\left(1-\cos \left(\omega_{1} t\right)\right)
$$

Thus, the expression of the simplified membrane model is similar, but of reduced form of that of the combined bending and membrane, in terms of the ODE constant $d_{3}$. This phase continues for a duration of $t=\tau$ when the loading is complete.

In the same spirit to the previous analyses, the dynamic equilibrium equation of the phase 2 leads to:

$$
\ddot{W}+\omega_{1}^{2} W=0
$$


which has a closed-form solution as $W=C_{3} \sin \left(\omega_{1} t\right)+C_{4} \cos \left(\omega_{1} t\right)$. Comparing the solution in this phase with the previous phase, while ensuring the kinematic admissibility at $t=\tau$ yields:

$$
\begin{gathered}
\ddot{W}_{2}=\frac{\eta H \omega_{1}^{2}}{2}\left[\cos \left(\omega_{1} t\right)-\cos \left(\omega_{1}(t-\tau)\right)\right] \\
\dot{W}_{2}=\frac{\eta H \omega_{1}}{2}\left(\sin \left(\omega_{1} t\right)-\sin \left(\omega_{1}(t-\tau)\right)\right) \\
W_{2}=\frac{\eta H}{2}\left(\cos \left(\omega_{1}(t-\tau)\right)-\cos \left(\omega_{1} t\right)\right)
\end{gathered}
$$

$$
\frac{W_{f}}{H}=\eta \sin \frac{\omega_{1} \tau}{2}
$$

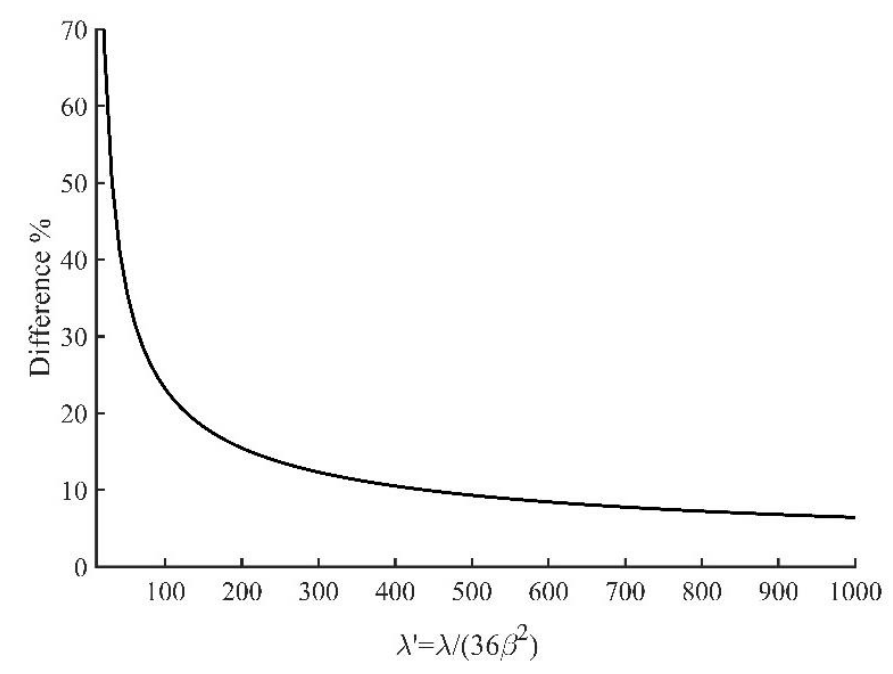

Fig. 19 The difference between the results of membraneonly and combined bending and membrane analyses

For high impulse magnitudes, the difference between the two approaches (combined bending and membrane and membrane only) decreases exponentially, the expression (52) yields about only $8 \%$ higher overestimation than its counterpart (Fig. 19).

\subsubsection{Pattern $(B)$ of deformation}

By compiling the energy equilibrium equation for pattern (B), in the first phase of motion, the 460 foregoing results in section4.1.1, i.e., the expressions (28)-(31) remain valid, while the succeeding 461 term $2 M_{0} /\left(1-\xi_{0}\right)$ in Eq. (31) vanishes, viz.:

$$
d_{4}=\frac{2 M_{0} \eta\left(\left(L b \xi_{0}+2\right) e^{-b\left(L \xi_{0}-r_{e}\right)}-(L b+2) \mathrm{e}^{-b\left(L-r_{e}\right)}+L b\left[\left(b^{2} r_{e}^{2}+2 b r_{e}+2\right)\left(\xi_{0}-1\right) / 2\right]\right)}{\beta\left(\xi_{0}-1\right) L^{3} b^{3}}
$$




$$
W_{1}=\frac{d_{4}}{B_{2}}\{\cos (\omega t)-1\}
$$

463

464

465

466

467

468

469

470

471

472

473

474

475

476

477

478

479

where $B_{2}$ is defined in Eq. (30). Thus, it is straightforward to show that the foregoing analyses in sections 4.2.1-4.2.2 remain valid throughout the first and second phases of motion,

The expressions for the displacement field and its time derivative may be merely obtained by replacing $d_{2}$ with $d_{4}$.

However, the ODE function describing the length of central plastic hinge line is now reduced to:

$$
f(t, \xi, \dot{\xi}): \frac{1}{6} \mu \omega H L^{2}(3 \xi+1)(\xi-1) \dot{\xi}-4 M_{0}(\xi+1)(\omega \sin (\omega \tau)(t-1)+1-\cos (\omega \tau))=0
$$

A time integration of Eq. (55), subsequent to the separation of variables finds the exact solution for the plastic hinge as:

$$
\begin{gathered}
\begin{array}{c}
F(t, \xi): 48 M_{0}\left(\omega \sin (\omega \tau)\left(\left(\frac{1}{2}\right) t^{2}-t\right)+t-\cos (\omega \tau) t\right) \\
=12 \mu \omega L^{2} H\left\{8 \ln (\xi+1)-10 \xi+3 \xi^{2}\right\}+C_{5}=0
\end{array} \\
C_{5}=(24 \tau \omega(\tau- \\
2) \sin (\omega \tau)-48 \tau \cos (\omega \tau)+48 \tau) M_{0} \\
+\left(-8 \mu \omega H L^{2} \ln \left(\xi_{0}+1\right)-\mu \omega H L^{2} \xi_{0}\left(3 \xi_{0}-10\right)\right)
\end{gathered}
$$

where the ODE constant $C_{5}$ is obtained by imposing the continuity of the closed form function $F\left(t=\tau, \xi=\xi_{0}\right)$ at the transition time point $t=\tau$. Since Eq. (55) is a closed-form expression, it is tractable analytically to obtain the end phase of motion. This phase terminates at $t=T_{2}$ as the length of the central plastic zone vanishes, i.e. $\xi=0$. Thus:

$$
T_{2}=(1-2 \tau) \mp \frac{\sqrt{6}}{12} \sqrt{24(3 \tau-1)^{2}-\left(\omega \tau M_{0}\right)^{-1} H L^{2} \mu\left(3 \xi_{0}^{2}+8 \ln \left(\xi_{0}+1\right)-10 \xi_{0}\right)}
$$

If $\xi_{0} \ll 1$, the terms of $\xi_{0}$ in the surd may be ignored, then $T_{2} \rightarrow \tau$ is suggestive of the solution approaches that of pattern (A) as the phase 1 and 2 merge together. While an exact solution to the Eq. (56) at $\xi(t)=0$ exists, for brevity in analysis, the evaluation of $T_{2}$ in Eq. (58) is approximated by truncating trigonometric terms into their equivalent Taylor series, i.e. $\sin \omega \tau \cong$ $\omega \tau, 1-\cos \omega \tau \cong \omega^{2} \tau^{2} / 2$.

$$
\frac{\dot{W}_{3}}{H \omega_{1}}=\frac{1}{2} \eta\left(\sin \left(T_{2} \omega_{1}\right)-\sin \left(\omega_{1}\left(T_{2}-\tau\right)\right)\right)-\frac{1}{2} \eta\left(\sin \left(\omega_{1} t\right)-\sin \left(\omega_{1}(t-\tau)\right)\right)-\frac{d_{4} \sin (\omega \tau)}{H \omega_{1} \omega A_{2}}
$$




$$
\begin{gathered}
\frac{W_{3}}{H}=\frac{1}{2} \omega_{1} \eta\left(T_{2}-t\right)\left\{\sin \left(T_{2} \omega_{1}\right)-\sin \left(\omega_{1}\left(T_{2}-\tau\right)\right)\right\} \\
+\frac{\eta}{2}\left\{\cos \left(\omega_{1}(t-\tau)\right)-\cos \left(\omega_{1}\left(T_{2}-\tau\right)\right)\right\}+\frac{1}{2} \eta\left[\cos \left(T_{2} \omega_{1}\right)-\cos \left(\omega_{1} t\right)\right] \\
+\frac{d_{4} \sin (\omega \tau)}{A_{2} \omega H}\left(T_{2}-t\right)-\frac{d_{4}\left\{\omega \sin (\omega \tau)\left(T_{2}-\tau\right)+1-\cos (\omega \tau)\right\}}{A_{2} \omega^{2}}
\end{gathered}
$$

481

It may be shown that the motion of the plate ceases at time $T_{f}$ expressed as:

$$
T_{f}=\frac{\left\{\omega_{1} \tau+2 \arccos \left(\cos \left(T_{2} \omega_{1}-\frac{\omega_{1} \tau}{2}\right)-\frac{d_{4} \sin (\omega \tau)}{H A_{2} \eta \omega_{1} \omega \sin \left(\frac{\omega_{1} \tau}{2}\right)}\right)\right\}}{2 \omega_{1}}
$$

482

483

\section{Impulsive loading}

484

A blast load of rectangular pulse shape with very short duration $(\tau \rightarrow 0)$ and very high 485 amplitude $\left(\eta \rightarrow \infty\right.$ or $\left.p_{1} \gg p_{c}\right)$ is known as impulsive loading. In the case of impulsive loading, the total change in linear momentum equals the total impulse imparted upon the system, hence the conservation of linear momentum implies that:

$$
\int_{0}^{\frac{r e}{L}} L^{2} \tau p_{0} z d z+\int_{\frac{r e}{L}}^{1} L^{2} \tau p_{0} a e^{-b L z} z d z=\int_{0}^{1} L^{2} \mu V_{0} z d z
$$

488

The solution to Eq. (62) yields:

$$
\begin{gathered}
V_{0}=\frac{\epsilon_{1} \tau p_{0}}{\mu} \\
\epsilon_{1}=\frac{r_{e}^{2} b^{2}+2 b r_{e}-2 a b L e^{-L b}-2 a e^{-L b}+2}{b^{2} L^{2}}
\end{gathered}
$$

The dimensionless kinetic energy is given as:

$$
\lambda=\frac{4 \mu V_{0}^{2} L^{2}}{\sigma_{0} H^{3} \epsilon_{1}^{2}}
$$

where $M_{0}=\sigma_{0} H^{2} / 4$ is assumed. When $r_{e} \rightarrow L$, and $\epsilon_{1} \rightarrow 1$, the expression for the impulsive velocity approaches to the uniform load available in the literature [36].

\subsection{Pattern (A)}

493

In the case of impulsive loading, the impact velocity is evaluated in terms of duration as $\tau=$ $494 \frac{\beta}{\eta \omega_{1}} \sqrt{24 \lambda}$ as $\sin \frac{\omega_{1} \tau}{2} \cong \frac{\omega_{1} \tau}{2}$. Eq. (30) then becomes: 


$$
\frac{W_{f}}{H}=\frac{1}{2}\left(\sqrt{\frac{24(\eta-1)}{\eta} \lambda \beta^{2}+1}-1\right)
$$

495 If the bending moment influence was discounted, using the approximation $\sin \frac{\omega_{1} \tau}{2} \cong \frac{\omega_{1} \tau}{2}$, the 496 permanent deformation can be recast in terms of the dimensionless kinetic energy as:

$$
\frac{W_{f}}{H}=\beta \sqrt{6 \lambda}
$$

497

when $r_{e} \rightarrow L$, and $\beta \rightarrow \frac{1}{6}$ the Eq. (66) converges to the solution found in the literature [36].

\section{$498 \quad 5.2 \quad$ Pattern (B)}

499 The impulsive load case of the plated elements undergoing the moving bending hinge may be 500 treated in the analogous manner as to the case of stationery plastic bending hinges. The 501 dimensionless kinetic energy may now be expressed as:

502

$$
\lambda=\left(\frac{\omega \tau \eta}{\beta}\right)^{2}\left\{\frac{-3 \xi_{0}^{3}+\xi_{0}^{2}+\xi_{0}+1}{1+\xi_{0}}\right\}
$$

The exact dynamic plastic curve, whereby the associated yield surface is characterised by the 504 parabola of Fig. 3, is bound between the inscribed and circumscribed curves as illustrated in Fig. 50520 . The solution to the inscribed yield criterion is recovered by replacing $\lambda$ with $\lambda / 0.618$ in the 506 expressions of each pattern. Clearly, the difference between the two curves is inconsequential 507 where the response is influenced by active bending hinge (Fig. 20b). However, for design 508 applications, given the charge mass and stand-off, both circumscribing and inscribing curves may 509 be implemented to estimate the response of the metal plates with a high degree of accuracy. 


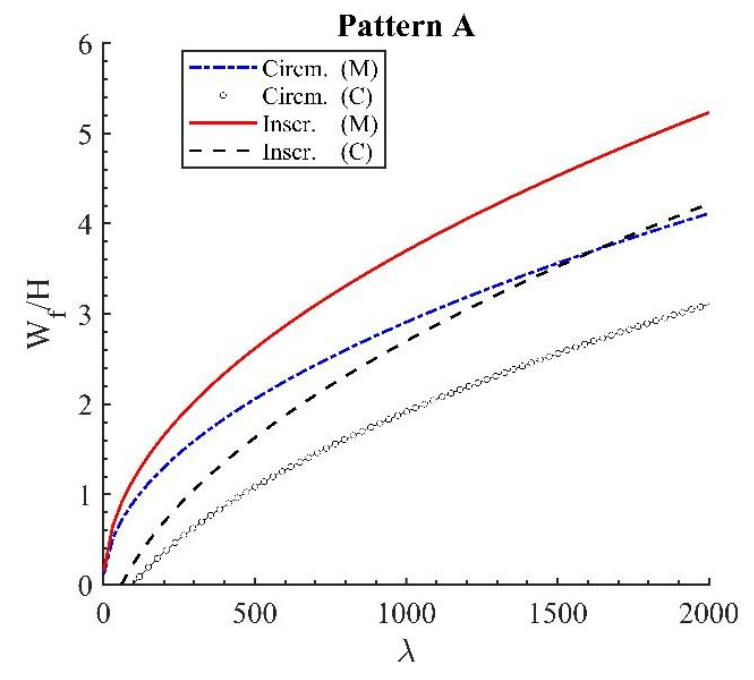

(a)

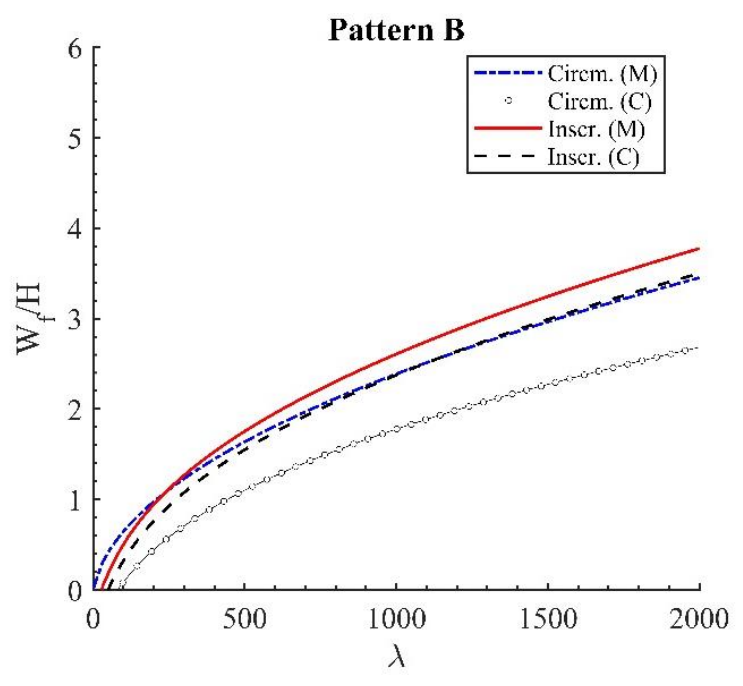

(b)

Fig. 20- Maximum permanent transverse displacements for various impulse values, with combined bending and membrane effects $(C)$, without bending effects $(M)$ comparison of (a) pattern $(A)$ and (b) pattern (B)

\subsection{Plates of various boundary conditions}

The dynamic plastic behaviour of panels which are secured with various boundary conditions

513 is obtained in a similar fashion to that of the simply supported case studied above. In such 514 circumstances, it is essential to consider the energy dissipated in the plastic hinge at the 515 boundaries corresponding to the fully clamped sides when formulating Eq. (15). This is given by $516 \alpha M_{0} \dot{\theta}_{2} L$, where $\alpha=1,2$ is the half the number of clamped faces. For example, for $\alpha=2$ the panels 517 are fully clamped, while for $\alpha=1$ only the two faces (typically the opposite sides) are clamped.

518 Thus, an additional term of $\alpha M_{0}$ would be added to Eq. (31). The deformation of first and second 519 phase of panels for pattern (A) would become:

$$
\begin{gathered}
W_{1}=\frac{1}{2} H\left(\eta-1-\frac{\alpha}{2}\right)\left(1-\cos \left(\omega_{1} t\right)\right) \\
W_{2}=-\frac{1}{2} H\left\{\left(\eta-\frac{\alpha}{2}-1\right) \cos \left(\omega_{1} t\right)-\eta \cos \left(\omega_{1}(t-\tau)\right)+\frac{\alpha}{2}+1\right\}
\end{gathered}
$$

520 Thus, the permanent deformation of the fully clamped, viz. impulsively loaded plate exhibiting stationery plastic hinge is furnished to

$$
\frac{W_{f}}{H}=\left(\frac{2 \bar{\lambda}}{3}+1\right)^{1 / 2}-1
$$


where $\bar{\lambda}=9 \beta^{2} \lambda$. Eq. (70) is similar to the expressions of fully clamped, uniformly loaded plate

523 found in the literature [36], except with the influence of the load shape appearing with coefficient $524 \beta$.

Similarly, for pattern (B) of motion, the expression (32) holds, but with $d_{2}$ replaced with $\bar{d}$ as:

$$
\begin{gathered}
\bar{d}=\frac{2 M_{0} \eta\left(\left(L b \xi_{0}+2\right) e^{-b\left(L \xi_{0}-r_{e}\right)}-(L b+2) \mathrm{e}^{-b\left(L-r_{e}\right)}+L b\left[\left(b^{2} r_{e}^{2}+2 b r_{e}+2\right)\left(\xi_{0}-1\right) / 2\right]\right)}{\beta\left(\xi_{0}-1\right) L^{3} b^{3}} \\
+\frac{(2+\alpha) M_{0}}{\left(\xi_{0}-1\right)}
\end{gathered}
$$

Eq. (71) indicates the ODE function $f(t, \xi, \dot{\xi})$ in (55) is increased by $\alpha M_{0}$. The permanent deformation due to the increased strain energy dissipated in the supports is given by:

$$
\begin{aligned}
W_{3}=\frac{1}{2} H \eta\left\{\cos \left(\omega_{1}(t-\tau)\right)-\cos \left(\omega_{1}\left(\bar{T}_{2}-\tau\right)\right)\right\} \\
+\frac{1}{4} H \omega_{1}\left(t-\bar{T}_{2}\right)\left[\sin \left(\omega_{1} \bar{T}_{2}\right)(\alpha+2-2 \eta)+2 \eta \sin \left(\omega_{1}\left(\bar{T}_{2}-\tau\right)\right)-4 \bar{d} \frac{\sin (\omega \tau)}{A_{2} H \omega \omega_{1}}\right] \\
+\frac{1}{4} H(\alpha+2-2 \eta)\left\{\cos \left(\omega_{1} t\right)-\cos \left(\omega_{1} \bar{T}_{2}\right)\right\} \\
+
\end{aligned}
$$

where $\bar{T}_{2}$ is the duration of the second phase of motion to be determined by numerical methods, in the same spirit as in Section 4.2.2.

\subsection{Comments on strain rate sensitivity (visco-plasticity)}

The scope of analysis in Sections 4 and 5 may be extended to encompass the strain-rate sensitivity of materials. Plastic hinges cannot develop in a strain rate sensitive material because an infinitely large strain would occur at a plastic hinge which would give rise to infinitely large stresses [20]. Thus, the result discussed here is a modal solution, similar to that of Jones [20] on the response of uniformly loaded circular and rectangular ductile metallic plates, but with the localised effects retained in the study.

Perrone and Bhadra [54] developed a method to approximate the influence of strain rate sensitivity using a mass connected by strings on either side. The system was loaded with uniform impulsive velocity $V_{1}=V_{0} / \epsilon$ to incur large inelastic deformation such that the influence of finite displacement was retained in the study. The authors observed that the maximum strain rate is reached when half of the kinetic energy is dissipated, i.e. at $V_{1} / \sqrt{2}$, at which point the transverse deformation reached two third of the permanent deformation. The strain rate was halved to estimate for the average strain rate. 
Using this approximation and considering the strain as $\varepsilon_{x} \cong(\partial w / \partial x)^{2} / 2$ or $\varepsilon_{y} \cong$ $545(\partial w / \partial y)^{2} / 2$ (ignoring the in-plane displacements), the strain rate, e.g. in zone I of pattern (A), is $546 \dot{\varepsilon}_{x} \cong W \dot{W} / L^{2}$, or $\dot{\varepsilon}_{x}=(1 / 2)\left(2 W_{f} / 3\right)\left(V_{1} / \sqrt{2}\right) / L^{2}$ and the average equivalent strain rate, assuming $547 \dot{\varepsilon}_{e} \cong\left(\varepsilon_{x}+\varepsilon_{y}\right) / \sqrt{3}$, is estimated by:

$$
\dot{\varepsilon}_{e} \cong \sqrt{2} W_{f} V_{1} /\left(3 L^{2}\right)
$$

548 Thus, assuming the plastic flow is governed by the Cowper-Symonds constitutive equation, the 549 dynamic yield stress is defined as follows:

$$
\sigma_{0}^{\prime}=\sigma_{0}\left(1+\left(\frac{\dot{\varepsilon_{e}}}{D}\right)^{1 / q}\right)
$$

where $\sigma_{0}{ }^{\prime}$ is the dynamic yield stress, $D$ and $q$ are the material constants obtained empirically using the Servo-Hydraulic machines or Hopkinson Bar strain rate gauges. The values of e.g. $q=5$ and $D=40.4 s^{-1}$ for mild steel or $q=5$ and $D=300 s^{-1}$ for RHA steel are most prevalent in the literature, although higher values of $D$ have also been reported on various low carbon steel and aluminium grades to accurately capture the deformations of the experimental/numerical models[6], [55], [56]. Since the parameter $q$ is quite large, even relatively rough estimates of strain rates would lead to reasonable predictions of the dynamic yield stress. Thus, the expression of equivalent strain rate may be implemented in Eq. (74). To avoid iterations, the expression of $W_{f}$ in Eq. (73) is replaced by its equivalent expression of dimensionless kinetic energy, i.e. either Eq. (66) for pattern (A) or using Eq. s' (67), and (60) for pattern (B). Thus, using the estimate $W_{f} / H=\sqrt{(2 / 3) \bar{\lambda}}$, the parameter $\sigma_{0}$ in the expressions of dimensionless kinetic energy may be replaced by $\sigma_{0}{ }^{\prime}$, although $\sigma_{0}$ is retained in Eq. (73) to avoid iterations. For example, for a simply supported plate, governed by roof shape velocity profile of pattern (A), we have $\dot{\varepsilon}_{e a}=$ $4 \beta V_{1}^{2} \sqrt{\left(\rho / \sigma_{0}\right)} / \sqrt{3} L$ thus:

$$
\frac{W_{f}}{H}=\left[\frac{2 \bar{\lambda}}{3\left(1+\left(\frac{4 \beta V_{1}^{2} \sqrt{\left(\rho / 3 \sigma_{0}\right)}}{D L}\right)^{1 / q}\right)}\right]^{1 / 2}
$$

\section{Validations of the theoretical models}

The accuracy of the proposed theoretical models is examined against the numerical and experimental studies using two approaches. In the first approach, a numerical model is set up to investigate the various cases of pattern (A) results (viz. membrane or combine bending and membrane). In the second category, the results of each pattern of motion were compared against 
the available experimental data on the effect of stand-off on blast phenomenon by Jacob et al [57], using mild steel plates, as well as those of Langdon et al. [58], [59] on blast protection armour steel plates. In addition, the numerical results of [60] are investigated for a comparative study. The experimental studies were conducted at Blast, Impact, and Survivability Research Unit of University of Cape Town. The difference between the experiments, despite the material type, geometry and load conditions presented in Table 2, was the method whereby the blast load was imparted on the panels. Jacob et al. [57] used a circular rigid-mild steel tube to provide the varying stand-off distance, hence confining the pressure to a radial central portion of the target plates, while Langdon et al. [58] utilised polystyrene bridge arrangement to provide the stand-off. Both studies employed PE4 disc explosive to generate the blast wave pressure. Details of these studies have been discussed elsewhere [58], [59], thus only the results of interest are presented here for validations.

\subsection{Materials and models}

In the current theoretical models, the influence of transverse shear and rotatory inertia have been ignored. This corresponds to a range of plate thicknesses as $H / L \leq 0.025$, a range pertinent to most practical design applications.

The candidate structures in the first approach were $400 \times 400 \mathrm{~mm}$ panels of ARMOX370T, ARMOX440T and Mild Steel (MS4), which material properties are presented in Table 1. ARMOX steel types are high strength RHA materials, fabricated by $\mathrm{SAAB}^{\circledR}$ and primarily designated for blast protection applications. These steel types exhibit high strength and hardness compared to mild steel but lower ductility, leading to less Specific Energy to Tensile Fracture (SETF). While mild steel material is moderately sensitive to strain rate, the armour steel materials are impervious to such phenomenological effects. As such, the aforementioned theoretical analyses are expected to yield good estimate for the response.

The candidate armour steel of [58], [60] were ARMOS370T, ARMOX 440T and ARMOX 500T. The panels had characteristic dimensions of $400 \mathrm{~mm} \times 400 \mathrm{~mm}$ (exposed area of $300 \mathrm{~mm} \times 300 \mathrm{~mm}$ ), and areal density of $29.8 \mathrm{~kg} / \mathrm{m}^{2}$ for AR $370 \mathrm{~T}$, while the AR440T had an areal density of $37 \mathrm{~kg} / \mathrm{m}^{2}$. The areal densities of MS4 and MS were 31.4 and $14.7 \mathrm{~kg} / \mathrm{m}^{2}$, respectively.

Fig. 21 draws the stress-strain curve of the armour steel specimen under uniaxial strain test. To account for the hardening of the materials, the mean flow stress for the analytical was averaged as $\sigma_{0}=\left(\sigma_{y}+\sigma_{U T}\right) / 2$. A loading decay parameter of $b=50 m^{-1}$, typical of most blast waves was taken for all blast scenarios studied here. 
Table 1- material properties of the steel panels (engineering stress values)

\begin{tabular}{ccccccc}
\hline \multirow{2}{*}{ Material } & $\begin{array}{c}\text { Designation/ } \\
\text { Mat. type }\end{array}$ & $\begin{array}{c}\boldsymbol{\sigma}_{\mathbf{0}} \\
(\mathbf{M P a})\end{array}$ & $\begin{array}{c}\boldsymbol{\sigma}_{U T} \\
(\mathbf{M P a})\end{array}$ & $\begin{array}{c}\mathbf{H} \\
(\mathbf{m m})\end{array}$ & $\begin{array}{c}\text { Hardness } \\
(\mathbf{H B W})\end{array}$ \\
\hline \multirow{2}{*}{ Ref. } & Armour & AR 370T & 1100 & 1270 & 3.81 & 380 \\
{$[58]-[60]$} & steel & AR 440T & 1210 & 1450 & 4.60 & 420 \\
& \multirow{2}{*}{ Mild Steel } & AR 500T & 1250 & 1470 & 4.15 & 480 \\
& MS4 & 330 & 400 & 4.00 & 120 \\
\hline Jacob et al. & Mild steel & MS & 240 & 356 & 1.90 & 120 \\
\hline
\end{tabular}

603

604

605

606

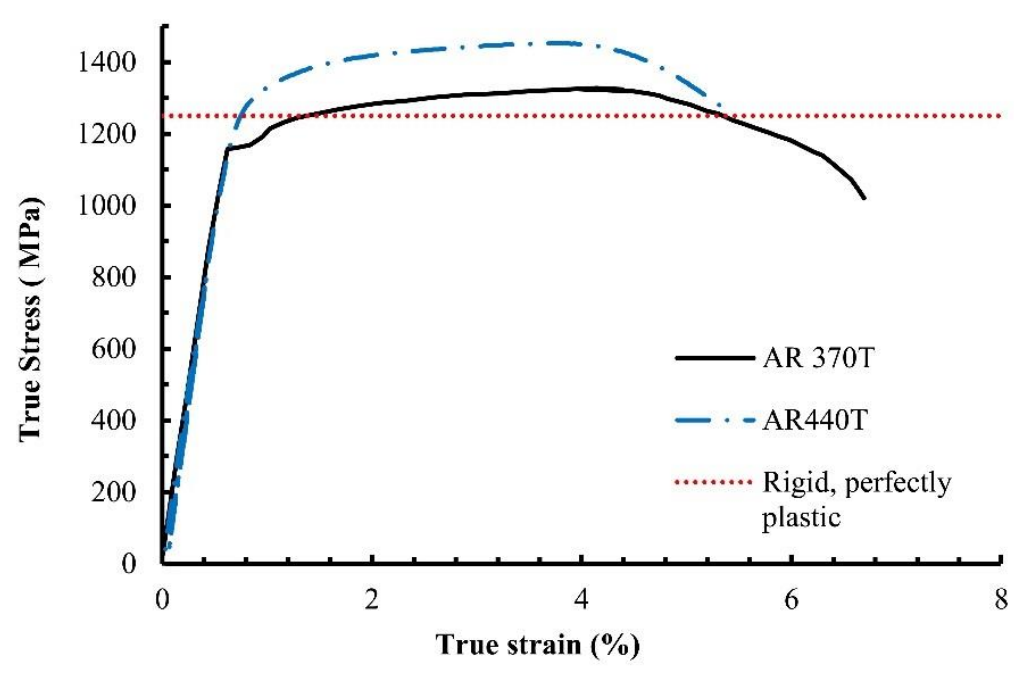

Fig. 21- Stress strain curve of armour steel models.

607

The experimental specimens of Jacob et al. had characteristic dimensions of $244 \mathrm{~mm} \times 244 \mathrm{~mm}$ 608 and $H / L=0.015$, while the loading was confined to the exposed area of $106 \mathrm{~mm}$ diameter.

\subsection{Finite Element (FE) model and validations}

610 A full 3D Finite Element model is set up in commercial software ABAQUS ${ }^{\circledR}$ Explicit 14.4 on 611 ARMOX steel and mild steel candidate materials. The blast load is axisymmetric and the plate has 6124 axes of symmetry. This reduced the size of numerical model to only a quarter of the plate with 613 associated symmetry boundary conditions as illustrated in Fig. 22. 
The Young modulus of the steel panels was assumed 200GPa and Poisson ratio as 0.3. The

615 modified Ramberg-Osgood constitutive model-with perfect plasticity- was assumed for the steel

616 materials. The strain rate sensitivity was investigated for mild steel only, as armour steels are in

617 general not very sensitive to strain rate. The panels were discretized with a mesh of four noded

618 S4R elements (doubly curved, generic shell elements with finite in-plane strain formulation)

619 having 5 Simpson points of integration through the plate thickness. S4R elements entail finite

620 membrane strains to capture bending and membrane strains, are of reduced integration and

621 hourglass control formulation. A total of 12550 elements were used (elemental length of $4 \mathrm{~mm}$ )

622 to satisfy mesh convergence. An additional $20 \mathrm{~mm}$ along the plate periphery was considered for

623 the two upper and lower clamps. The clamps were tied to the plate by penalty contact of

624 coefficient 0.3 and modelled as rigid body.

The loading was implemented by a FORTRAN coded user defined subroutine VDLOAD in each case. To maintain low ratio of loading duration to the natural period of the plate, a rectangular pulse shape profile with low duration $(\tau=50 \mu s)$ was assumed for the pulse shape. With respect

628 to the spatial distribution of the load, the radius of the centrally blast loaded plate was calculated 629 as $100 \mathrm{~mm}$ and $50 \mathrm{~mm}$ for AR440T and AR370T, respectively. The magnitude of the load varied in 630 the range of 10-70MPa, as shown in Fig. 23, which corresponds of a range of blast loads with 631 various stand-off distances for the same charge mass. Using the procedure to determine the 632 impulsive asymptote (simplified model), the results of membrane/combined bending and 633 membrane cases are compared in Table 3. In the case of $\omega_{0} \rightarrow 1$ the results concur with the 634 uniform loading case by [36].

635 The mild steel panels, on the other hand, had various loading constant zone radii, while the 636 magnitude of the load was adjusted to yield constant total impulse of 50N.s. Thus, using the 637 expression $I=\iint p(x, y, t) d x d y d t$, a crude estimate for the calculated pressure was found as $638 p_{0}=I /\left(\pi r_{e}^{2} \tau\right)$. Subsequently, each considered load parameter $\omega_{0}=[0.25,0.5,0.75,1]$ 639 corresponds to pressure of $p_{0}=[127,34.8,14.1,7.96] M P a$, respectively. 


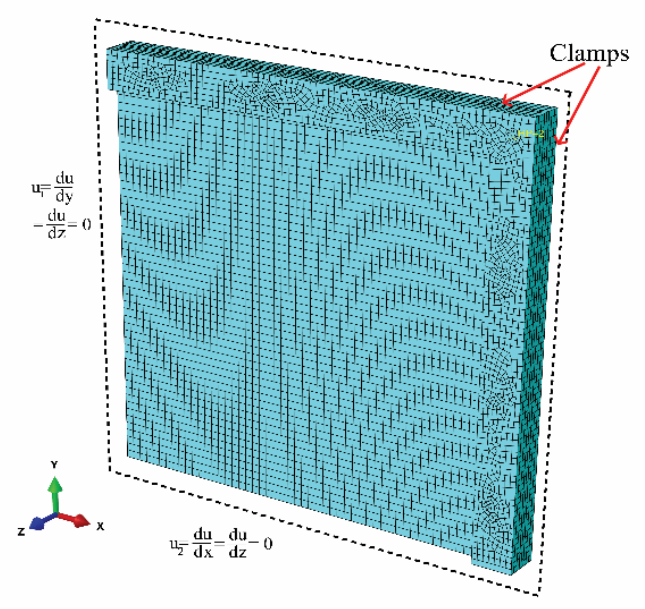

Fig. 22- FE mesh of the quarter plate model

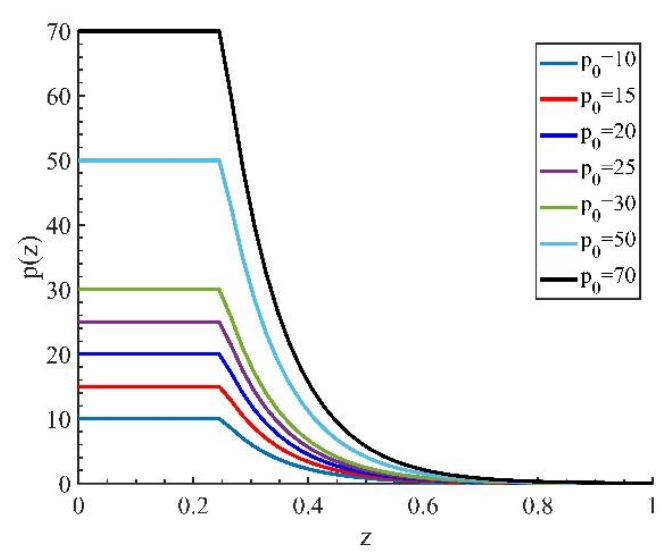

Fig. 23- Spatial distribution of the blast loads (central intersection view)

640

641 It turns out that the theoretical results on Armour panels concur with the numerical results, 642 with a maximum average difference occurring at $p_{0}=20 \mathrm{MPa}$ as $22 \%$ and $19.5 \%$ for AR370T and 643 AR440T, respectively. The membrane formulation only overpredicts the results when the 644 magnitude of pressure is low, as in such circumstances the contribution from bending moments 645 the overall response is significant and shall be retained in the study. Referring to the strain rate 646 sensitivity analyses on mild steel, Perrone and Bhadra's estimate with $D=40.4$ yields maximum 647 of $23 \%$ larger displacement on $\omega_{0}=0.5$ (Fig. 24).

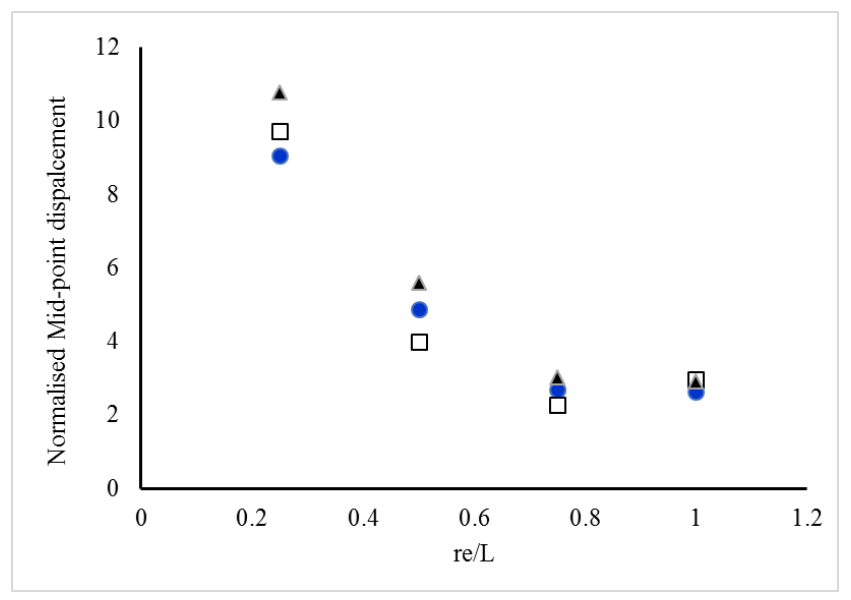

Fig. 24-comparison of numerical and theoretical results (Eq. (75)) on MS4 panels, $\square$ Numerical results, theoretical results with $D=40.4 s^{-1}$ and $q=5$, theoretical results with $D=1300 s^{-1}$ and $q=5$ 
AR440T

\begin{tabular}{|c|c|c|c|c|c|c|c|c|c|c|c|c|}
\hline \multirow[b]{2}{*}{$p_{0}$} & \multirow[b]{2}{*}{$\lambda$} & \multicolumn{2}{|c|}{$W_{f} / H(C)$} & \multicolumn{2}{|c|}{$W_{f} / H(\mathbf{M})$} & \multirow[b]{2}{*}{ ABAQUS } & \multirow[b]{2}{*}{$\lambda$} & \multicolumn{2}{|c|}{$W_{f} / H(C)$} & \multicolumn{2}{|c|}{$W_{f} / H(M)$} & \multirow[b]{2}{*}{ ABAQUS } \\
\hline & & $\begin{array}{l}\text { Full } \\
\text { Eq. }\end{array}$ & Simpl. & $\begin{array}{c}\text { Full } \\
\text { Eq. }\end{array}$ & Simpl. & & & $\begin{array}{c}\text { Full } \\
\text { Eq. }\end{array}$ & Simpl. & $\begin{array}{c}\text { Full } \\
\text { Eq. }\end{array}$ & Simpl. & \\
\hline 10 & 9.32 & 0.389 & 0.492 & 0.797 & 0.805 & 0.996 & 21.25 & 0.19 & 0.39 & 0.54 & 0.54 & 0.192 \\
\hline 15 & 20.98 & 0.740 & 0.787 & 1.196 & 1.208 & 0.718 & 47.81 & 0.40 & 0.50 & 0.81 & 0.82 & 0.793 \\
\hline 20 & 37.30 & 1.113 & 1.135 & 1.595 & 1.610 & 1.222 & 85.00 & 0.63 & 0.69 & 1.08 & 1.09 & 0.964 \\
\hline 25 & 58.27 & 1.496 & 1.503 & 1.994 & 2.013 & 1.763 & 132.81 & 0.88 & 0.92 & 1.35 & 1.36 & 1.167 \\
\hline 30 & 83.91 & 1.885 & 1.882 & 2.392 & 2.416 & 2.504 & 191.25 & 1.13 & 1.16 & 1.62 & 1.63 & 1.280 \\
\hline 50 & 233.09 & 3.459 & 3.439 & 3.987 & 4.026 & 4.396 & 531.25 & 2.18 & 2.17 & 2.69 & 2.72 & 2.585 \\
\hline 70 & 456.87 & 6.045 & 5.020 & 5.582 & 5.637 & 5.43 & 1041.25 & 3.24 & 3.23 & 3.77 & 3.80 & 4.176 \\
\hline
\end{tabular}

\subsection{Discussion of the results and of experimental validations}

In Fig. 25 we compare the analytical models of pattern (A) and (B) with the results of Jacob et al. [57]. The radius of the blast $r_{e}$ is estimated as the charge radius. Under the circumstances where the charge radius to plate length ratio is more than a third, pattern (B) gives a more conservative estimate in accordance with the experimental values. Clearly, the prediction of the permanent displacement necessitates the accurate prediction of the load radius and exponent from the experimentally/numerically captured pressure time histories, or using empirical estimates [1]. The pressure loads of shorter stand-off result in higher values of permanent deformation, evidently as most of the blast is concentrated on the localised region of the target.

664 Due to the proximity of the blast the ratio of $r_{e} / L$ decreases, while the decay exponent increases.

665 However, the length of the target plate is restrained to the portion to which most of the blast load 666 is absorbed. This can be estimated as the exposed area of the plate.

Under the circumstances where the blast pressure is induced by a proximal charge and the ratio of the charge diameter to the expensed length of the target is small, such as those in [60]

669 (Fig. 26), the majority of the load is dissipated before reaching the target boundaries, thus, it is 670 reasonable to consider the effective length of the panels in use of pattern (A). The analytical 671 studies are compared against the exposed area of $300 \times 300 \mathrm{~mm}$ target where in the range of $672 r_{e} / L \ll 1$, pattern (A) gives an upper bound prediction while the results of pattern (B) are too 
675

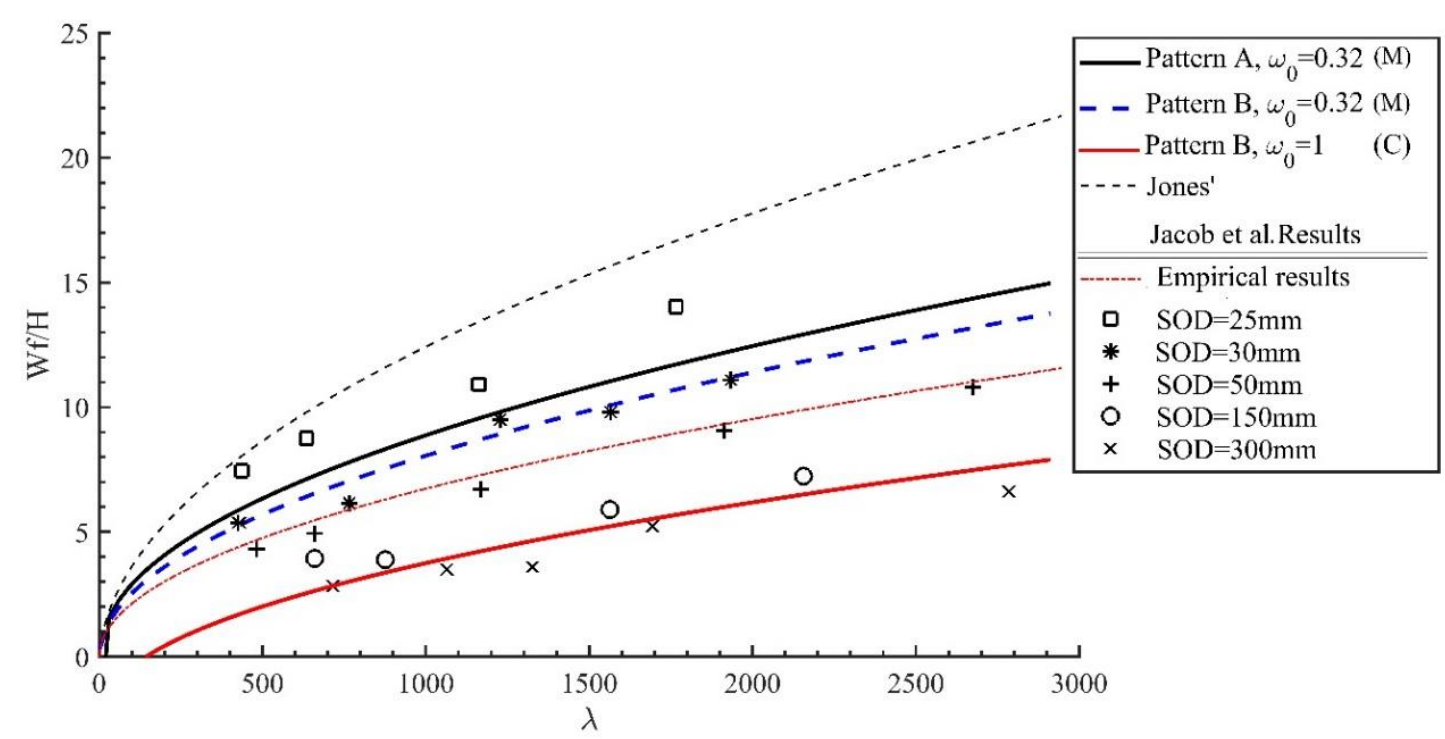

Fig. 25- predicted curves of the permanent deformation due to membrane effect (circumscribing yield criterion), compared against the experimental data by Jacob et al.

An odd result of [57] in Fig. 25 was that while the blast load may be assumed as uniform with increased stand-off, such results deviated from the predicted theoretical values in the literature.

678 This is because the large strain localisations due to the proximity of blast would lead to adiabatic 679 shear deformation due to the high temperature. The adiabatic heat generated due to the high 680 strain rates leads to the elevated temperature in the localised region while the surrounding 681 region of the plate strain hardens. In such cases the thermal softening may overcome the strain 682 hardening effects. Thus, the mathematical treatment incorporating the adiabatic shear effects is fraught with difficulty, and to date the authors are unaware of an exact solution where this effect was retained in the analyses of this kind. However, the concurrence of the, herein, theoretical approach and the available experimental/ numerical results in the literature is promising. 


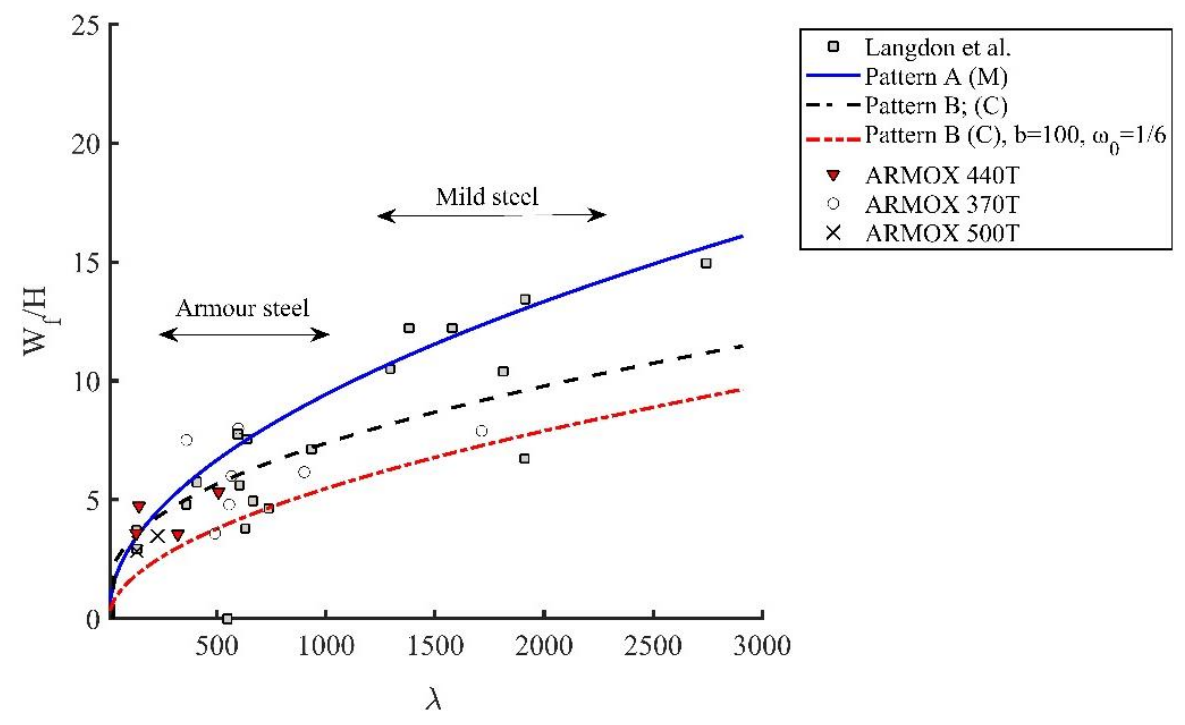

Fig. 26- Prediction of the permanent deformation, theoretical models vs. the experimental data of Langdon et al. [58]

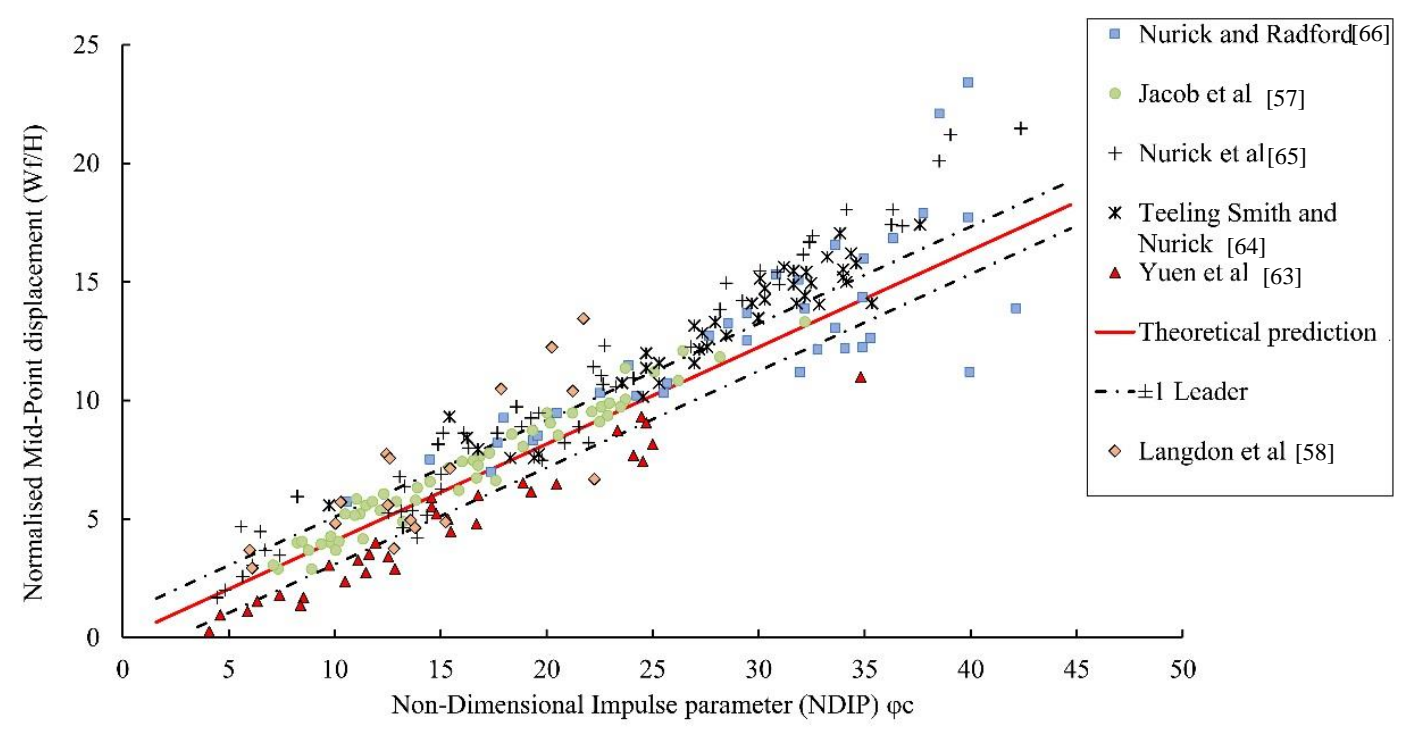

Fig. 27- Theoretical predictions of deformation of the square plate (membrane only) with $\beta=1 / 12$ and $b=50 m^{-1}$ (typical values of blast wave) compared against the experimental data by researchers post 1989.

687 In Fig. 27, the solution of membrane case is compared against the experiments post 1989. The 688 curve is casts into Non-Dimensional Impulse Parameter (NDIP) $\phi_{c}=\sqrt{\lambda} / 2$ and a critical estimate 689 of $\beta=1 / 12$ was considered. 


\section{Conclusions}

692 This paper deals with a rigorous analytical study to predict the nonlinear dynamic 693 performance of thin square plates with various boundary conditions. The armour plates were 694 made of strain rate insensitive, rigid-perfectly plastic ductile metals with isotropic hardening 695 behaviour, upon which a localised blast pressure pulse is imparted laterally.

696 Such a blast emanates from detonation of explosives having arbitrary mass and proximity to 697 the target. The blast wave of this kind is represented by a spatial and a temporal distribution. The 698 spatial distribution was characterised by means of an axisymmetric, piecewise continuous 699 function in polar coordinates, having constant pressure over the central zone, followed by an 700 exponentially decaying function on the remainder of this zone. Each of the blast parameters of the spatial distribution have been found as bijective functions of the stand-off distance and the explosive mass [1], [53]. The loading type is, therefore, universal which enables the theoretical solution capable of modelling close-in to uniform blast load responses.

The pulse shape effects have not been studied here. This is because the method of eliminating the pulse shape proposed by Youngdahl has well been examined in the literature [24], [46], [48], [50], [61]. The difference between the effect of pulse shapes of rectangular and linear functions for circular plate was found to be 5\% [41]. Thus, the permanent transverse deflection may be furnished using the impulsive simplification for the assumed rectangular pulse, irrespective of the pulse shape. For blast of high magnitude and low pulse duration, the transverse deformation was reduced into an expression of dimensionless initial kinetic energy and found to be consistent with the available experimental results in the literature.

The plates were assumed as 'thin' membranes, indicating the influence of transverse shear and rotatory inertia (corresponding to the Mindlin-Reissner plate theory) could be disregarded. With large deflection theory in mind, the influence of the geometry changes, or finite displacements were retained in the analyses. This reflects on the roof shaped deformation profile having stationery or travelling plastic hinges, which latter results yield more conservative estimates than the former.

A final remark seems in order. While the results are most suitable for rate insensitive materials, such as high strain ARMOX steel, the results prove to be conservative when implemented on the materials which exhibit visco-plasticity (strain rate sensitivity) effects. However, using the modal method proposed by researchers [20], Perrone and Bhadra's approximation and Cowper-Symonds equation, the commentary on the response of rate sensitive materials has been discussed in the context of the problem. The current results in each case may 


\section{References}

[1] N. Mehreganian, A. S. Fallah, G. K. Boiger, and L. A. Louca, "RESPONSE OF ARMOUR STEEL SQUARE PLATES TO LOCALISED AIR BLAST LOAD- A DIMENSIONAL ANALYSIS,” Int. J. Multiphys., no. December, pp. 1-20, 2017.

[2] G. N. Nurick and V. . Balden, "Localised Blast Loaded Circular Plates: an Experimental and Numerical Investigation," Proc. IMPLAST 2010 Conf., vol. 12, no. 14, pp. 145-152, 2010.

[3] D. Karagiozova, G. S. Langdon, G. N. Nurick, and S. Chung Kim Yuen, "Simulation of the response of fibre-metal laminates to localised blast loading," Int. J. Impact Eng., vol. 37, no. 6, pp. 766-782, 2010.

[4] G. S. Langdon, S. C. K. Yuen, and G. N. Nurick, "Experimental and numerical studies on the response of quadrangular stiffened plates. Part II: Localised blast loading," Int. J. Impact Eng., vol. 31, no. 1, pp. 85-111, 2005.

[5] G. N. Nurick and J. B. Martin, "Deformation of thin plates subjected to impulsive loading-A review," Int. J. Impact Eng., vol. 8, no. 2, pp. 159-170, Jan. 1989.

[6] N. Jacob, S. C. K. Yuen, G. N. Nurick, D. Bonorchis, S. A. Desai, and D. Tait, "Scaling aspects of quadrangular plates subjected to localised blast loads - Experiments and predictions," Int. J. Impact Eng., vol. 30, no. 8-9, pp. 1179-1208, 2004.

[7] D. Bonorchis and G. N. Nurick, "The effect of welded boundaries on the response of rectangular hot-rolled mild steel plates subjected to localised blast loading," Int. J. Impact Eng., vol. 34, no. 11, pp. 1729-1738, 2007.

[8] T. Børvik, A. G. Hanssen, M. Langseth, and L. Olovsson, "Response of structures to planar blast loads - A finite element engineering approach," Comput. Struct., vol. 87, no. 9-10, pp. 507-520, 2009.

[9] S. Dey, T. Børvik, X. Teng, T. Wierzbicki, and O. S. Hopperstad, "On the ballistic resistance of double-layered steel plates: An experimental and numerical investigation,” Int. J. Solids Struct., vol. 44, no. 20, pp. 6701-6723, 2007.

[10] V. Aune, E. Fagerholt, K. O. Hauge, M. Langseth, and T. Børvik, "Experimental study on the response of thin aluminium and steel plates subjected to airblast loading," Int. J. Impact Eng., vol. 90, pp. 106-121, 2016.

[11] V. Aune, G. Valsamos, F. Casadei, M. Larcher, M. Langseth, and T. Børvik, "Numerical study on the structural response of blast-loaded thin aluminium and steel plates," Int. J. Impact Eng., vol. 99, pp. 131-144, 2017.

[12] H. G. Hopkins and W. Prager, "The Load carrying capacity of circular plates," J. Mech. nd Phys. Solids, vol. 2, no. June, pp. 1-18, 1953.

[13] A. D. Cox and L. W. Morland, "Dynamic plastic deformations of simply-supported square plates," J. Mech. Phys. Solids, vol. 7, no. March 1959, pp. 229-241, 1959.

[14] K. L. Komarov and Y. V. Nemirovskii, "Dynamic behaviour of rigid-plastic rectangular plates (Translated)," Prikl. Mekhanika, vol. 21, no. 7, pp. 683-690, 1986.

[15] N. Jones and J. Gomes De Oliveira, "The Influence of Rotatory Inertia and Transverse Shear on the Dynamic Plastic Behavior of Beams," J. Appl. Mech., vol. 46, no. June 1979, pp. 303-310, 1979.

[16] N. Jones and J. Gomes De Oliveira, "Dynamic Plastic Response of Circular Plates With Transverse Shear and Rotatory Inertia,” J. Appl. Mech., vol. 47, no. March 1980, pp. 27-34, 1980.

[17] E. Onat and R. Haythornthwaite, "The load carrying capacity of circular plates at large deflection," 1954.

[18] F. L. Chen and T. X. Yu, "Membrane Factor Method for large deflection response of beams and plates to intense dynamic loading," vol. 141, pp. 59-71, 2014.

[19] N. Jones and R. . Walters, "Large deflections of rectangular plates," J. Sh. Res., vol. 18, no. 2, pp. 125-131, 1983.

[20] N. Jones, "Dynamic inelastic response of strain rate sensitive ductile plates due to large impact, dynamic pressure and explosive loadings," Int. J. Impact Eng., vol. 74, pp. 3-15, 2014.

[21] N. Jones, "A theoretical study of the dynamic plastic behaviour of beams and plates with finite-deflections," Int. J. Solids Struct., vol. 7, no. 33, pp. 1007-1029, 1971.

[22] N. Jones, "Combined distributed loads on rigid-plastic circular plates with large deflections," Int. J. Solids Struct., vol. 5, no. 1, pp. 51-64, 1969.

[23] Z. Xue and J. W. Hutchinson, "A comparative study of impulse-resistant metal sandwich plates," Int. J. Impact Eng., vol. 30, no. 10, pp. 1283-1305, 2004.

[24] Y. Yuan and P. J. Tan, "Deformation and failure of rectangular plates subjected to impulsive loadings," Int. J. Impact Eng., vol. 59, pp. 46-59, 2013.

[25] G. S. Langdon and G. K. Schleyer, "Inelastic deformation and failure of profiled stainless steel blast wall panels. Part II: Analytical modelling considerations," Int. J. Impact Eng., vol. 31, no. 4, pp. 371-399, 2005.

[26] G. S. Langdon and G. K. Schleyer, "Inelastic deformation and failure of profiled stainless steel blast wall panels. Part I: Experimental investigations," Int. J. Impact Eng., vol. 31, no. 4, pp. 341-369, 2005.

[27] E. Nwankwo, A. Soleiman Fallah, G. S. Langdon, and L. A. Louca, "Inelastic deformation and failure of partially strengthened profiled blast walls,” Eng. Struct., vol. 46, pp. 671-686, 2013.

[28] E. Nwankwo, A. Soleiman Fallah, and L. A. Louca, "An investigation of interfacial stresses in adhesively-bonded single lap joints subject to transverse pulse loading," J. Sound Vib., vol. 332, no. 7, pp. 1843-1858, 2013.

[29] Q. M. Li, "Continuity conditions at bending and shearing interfaces of rigid, perfectly plastic structural elements," Int. J. Solids Struct., vol. 37, no. 27, pp. 3651-3665, 2000.

[30] W. Q. Shen and N. Jones, “A failure criterion for beams under impulsive loading,” Int. J. Impact Eng., vol. 12, no. 
[31] C. Zheng, X. Kong, W. Wu, and F. Liu, "The elastic-plastic dynamic response of stiffened plates under confined blast load," Int. J. Impact Eng., vol. 95, pp. 141-153, 2016.

[32] M. Toolabi, A. S. Fallah, P. . Baiz, and L. . Louca, "Enhanced mixed interpolation XFEM formulations for discontinuous Timoshenko beam and Mindlin-Reissner plate," Int. J. Numer. Methods Eng., no. April 2018, pp. 124, 2018.

[33] N. Jones, T. O. Uran, and S. A. Tekin, "The dynamic plastic behavior of fully clamped rectangular plates," Int. J. Solids Struct., vol. 6, no. 12, pp. 1499-1512, 1970.

[34] Wierzbicki T and Florence AL, "Theoretical and Experimental Investigation of Impulsively Loaded Clamped Circular Viscoplastic Plates,” Int. J. Solids Struct., vol. 6, no. 5, pp. 553-568, 1970.

[35] N. Jones, "Impact loading of ductile rectangular plates," Thin-Walled Struct., vol. 50, no. 1, pp. 68-75, 2012.

[36] N. Jones, Structural Impact, 1st ed. Cambridge: Cambridge University Press, 1997.

[37] T. X. Yu and F. L. Chen, "The large deflection dynamic plastic response of rectangular plates," Int. J. Impact Eng., vol. 12 , no. 4, pp. 605-616, 1992.

[38] P. S. Symonds and N. Jones, "Impulsive loading of fully clamped beams with finite plastic deflections and strainrate sensitivity," Int. J. Mech. Sci., vol. 14, no. 1, pp. 49-69, 1972.

[39] K. Micallef, A. S. Fallah, D. J. Pope, and L. A. Louca, "Dynamic Performance of Simply Supported Rigid Plastic Circular Thick Steel Plates Subjected to Localized Blast Loading,” J. Eng. Mech., no. January, pp. 159-171, 2014.

[40] T. . Yu and F. L. Chen, "The large deflection dynamic plate response of rectangular plates," Int. J. Impact Eng., vol. 12, no. 4, pp. 603-616, 1992.

[41] K. Micallef, A. S. Fallah, P. T. Curtis, and L. A. Louca, "On the dynamic plastic response of steel membranes subjected to localised blast loading,” Int. J. Impact Eng., vol. 89, pp. 25-37, 2016.

[42] Y. Yuan, P. J. Tan, K. A. Shojaei, and P. Wrobel, "Large deformation, damage evolution and failure of ductile structures to pulse-pressure loading,” Int. J. Solids Struct., vol. 96, pp. 320-339, 2016.

[43] N. Jones, "The credibility of predictions for structural designs subjected to large dynamic loadings causing inelastic behaviour," Int. J. Impact Eng., vol. 53, pp. 106-114, 2013.

[44] D. Bonorchis and G. N. Nurick, "The influence of boundary conditions on the loading of rectangular plates subjected to localised blast loading - Importance in numerical simulations," Int. J. Impact Eng., vol. 36, no. 1, pp. 40-52, 2009.

[45] D. Karagiozova, T. X. Yu, and G. Lu, "Transverse blast loading of hollow beams with square cross-sections," ThinWalled Struct., vol. 62, pp. 169-178, 2013.

[46] K. Micallef, a. S. Fallah, D. J. Pope, and L. A. Louca, "The dynamic performance of simply-supported rigid-plastic circular steel plates subjected to localised blast loading," Int. J. Mech. Sci., vol. 65, no. 1, pp. 177-191, 2012.

[47] A. S. Fallah, K. Micallef, G. S. Langdon, W. C. Lee, P. T. Curtis, and L. A. Louca, "Dynamic response of Dyneema ${ }^{\circledR}$ HB26 plates to localised blast loading," Int. J. Impact Eng., vol. 73, pp. 91-100, 2014.

[48] C. K. Youngdahl, "Correlation parameters for eliminating the effects of pulse shape on dynamic plate deformation," Trans. ASME J. Appl. Mech., vol. 37, no. 2, pp. 744-752, 1970.

[49] C. K. Youngdahl, "Influence of pulse shape on the final plastic deformation of a circular plate," Int. J. Solids Struct., vol. 7, no. 9, pp. 1127-1142, 1971 .

[50] Q. M. Li and N. Jones, "Foundation of Correlation Parameters for Eliminating Pulse Shape Effects on Dynamic Plastic Response of Structures,” J. Appl. Mech., vol. 72, no. 2, p. 172, 2005.

[51] Q. M. Li and Y. G. Huang, "Dynamic plastic response of thin circular plates with transverse shear and rotatory inertia subjected to rectangular pulse loading," Int. J. Impact Eng., vol. 8, no. 3, pp. 219-228, 1989.

[52] K. Micallef, "The dynamic response of blast-loaded monolithic and composite plated structures: A thesis submitted for the degree of Doctor of Philosophy ( Ph . D .),” Imperial College London, 2013.

[53] K. Micallef, A. Soleiman Fallah, D. J. Pope, M. Moatamedi, and L. A. Louca, "On dimensionless loading parameters for close-in blasts,” Int. Jounral Multiphysics, vol. 9, no. 2, pp. 171-194, 2015.

[54] N. Perrone and P. Bhadra, "A Simplified Method to Account for Plastic Rate Sensitivity With Large Deformations," J. Appl. Mech., vol. 46, no. 4, p. 811, 1979.

[55] A. Neuberger, S. Peles, and D. Rittel, "Springback of circular clamped armor steel plates subjected to spherical airblast loading," Int. J. Impact Eng., vol. 36, no. 1, pp. 53-60, 2009.

[56] G. N. Nurick and J. B. Martin, "Deformation of thin plates subjected to impulsive loading-a review -Part I: Theoretical considerations," Int. J. Impact Eng., vol. 8, no. 2, pp. 159-170, 1989.

[57] N. Jacob, G. N. Nurick, and G. S. Langdon, "The effect of stand-off distance on the failure of fully clamped circular mild steel plates subjected to blast loads,” Eng. Struct., vol. 29, no. 10, pp. 2723-2736, 2007.

[58] G. S. Langdon, W. C. Lee, and L. A. Louca, "The influence of material type on the response of plates to air-blast loading,” Int. J. Impact Eng., vol. 78, pp. 150-160, 2015.

[59] R. J. Curry and G. S. Langdon, "Transient response of steel plates subjected to close proximity explosive detonations in air," Int. J. Impact Eng., vol. 102, pp. 102-116, 2017.

[60] N. Mehreganian, L. A. Louca, G. S. Langdon, R. J. Curry, and N. Abdul-Karim, "The response of mild steel and armour steel plates to localised air-blast loading-comparison of numerical modelling techniques," Int. J. Impact Eng., vol. 115, no. May, pp. 81-93, 2018.

[61] Q. M. Li and N. Jones, "Blast loading of fully clamped circular plates with transverse shear effects," Int. J. Solids Struct., vol. 31, no. 14, pp. 1861-1876, 1994.

[62] G. R. Johnson and W. H. Cook, "Fracture characteristics of three metals subjected to various strains, strain rates, temperatures and pressures," Eng. Fract. Mech., vol. 21, no. 1, pp. 31-48, 1985.

[63] S. Chung Kim Yuen, G. N. Nurick, G. S. Langdon, and Y. Iyer, "Deformation of thin plates subjected to impulsive 
load: Part III - an update 25 years on,” Int. J. Impact Eng., vol. 107, pp. 1339-1351, 2017.

[64] R. G. Teeling-Smith and G. N. Nurick, "The deformation and tearing of thin circular plates subjected to impulsive loads," Int. J. Impact Eng., vol. 11, no. 1, pp. 77-91, 1991.

[65] T. J. Cloete, G. N. Nurick, and R. N. Palmer, "The deformation and shear failure of peripherally clamped centrally supported blast loaded circular plates," Int. J. Impact Eng., vol. 32, no. 1-4, pp. 92-117, 2006.

[66] G. N. Nurick and A. M. Radford, "Deformation and tearing of clamped circular plates subjected to localised central blast loads," Recent Dev. Comput. Appl. Mech. (Ed. B.D Reddy), vol. a volume i, pp. 276-301, 1997. 